\title{
Self-organization, quality control, and preclinical studies of human iPSC-derived retinal sheets for tissue-transplantation therapy
}

Kenji Watari ${ }^{1, \S}$, Suguru Yamasaki ${ }^{1,2, \S}$, Hung-Ya Tu², Masayuki Shikamura ${ }^{3}$, Tatsuya Kamei $^{1}$, Hideki Adachi ${ }^{4}$, Tomoaki Tochitani ${ }^{4}$, Yasuyuki Kita ${ }^{1}$, Aya Nakamura ${ }^{5}$, Kazuki Ueyama $^{5}$, Keiichi Ono ${ }^{5}$, Chikako Morinaga ${ }^{2,6}$, Take Matsuyama ${ }^{2}$, Junki Sho ${ }^{2}$, Miyuki Nakamura $^{3}$, Masayo Fujiwara ${ }^{1}$, Yoriko Hori ${ }^{1}$, Anna Tanabe ${ }^{1}$, Rina Hirai ${ }^{1}$, Orie Terai ${ }^{1}$, Osamu Ohno ${ }^{1}$, Hidetaka Ohara ${ }^{1}$, Tetsuya Hayama ${ }^{1}$, Atsushi Ikeda ${ }^{1}$, Daiki Nukaya ${ }^{1}$, Keizo Matsushita ${ }^{1,2}$, Masayo Takahashi ${ }^{2}$, Akiyoshi Kishino ${ }^{1}$, Toru Kimura ${ }^{1}$, Shin Kawamata $^{3}$, Michiko Mandai ${ }^{2,6}$, and Atsushi Kuwahara ${ }^{1, *}$

${ }^{1}$ Regenerative \& Cellular Medicine Kobe Center, Sumitomo Dainippon Pharma Co., Ltd., Chuo-ku, Kobe 650-0047, Japan;

${ }^{2}$ Laboratory for Retinal Regeneration, RIKEN Center for Biosystems Dynamics Research, Chuo-ku, Kobe 650-0047, Japan;

${ }^{3}$ Research \& Development Center for Cell Therapy, Foundation for Biomedical Research and Innovation at Kobe, Chuo-ku, Kobe 650-0047, Japan;

${ }^{4}$ Preclinical Research Unit, Research Division;

${ }^{5}$ Technology Research \& Development Division,

Sumitomo Dainippon Pharma Co., Ltd., Chuo-ku, Kobe 650-0047, Japan;

${ }^{6}$ RIKEN Program for Drug Discovery and Medical Technology Platforms, RIKEN

Cluster for Science, Technology and Innovation Hub., Saitama 351-0198, Japan;

$\S$ These authors contributed equally to this work.

*To whom correspondence should be addressed:

Atsushi Kuwahara, Ph.D.

Regenerative \& Cellular Medicine Kobe Center,

Sumitomo Dainippon Pharma Co., Ltd.

1-5-2 Minatojima Minamimachi, Chuo-ku, Kobe, Hyogo 650-0047, Japan

TEL +81-78-306-2170

E-mail: atsushi-kuwahara@ds-pharma.co.jp 


\begin{abstract}
Three-dimensional retinal organoids (3D-retinas) are a promising graft source for transplantation therapy. We previously developed self-organizing culture for 3D-retina generation from human pluripotent stem cells (hPSCs). Here we present a quality control method and preclinical studies for tissue-sheet transplantation. Self-organizing hPSCs differentiated into both retinal and off-target tissues. Gene expression analyses identified the major off-target tissues as eye-related, cortex-like, and spinal cord-like tissues. For quality control, we developed a qPCR-based test in which each hPSCderived neuroepithelium was dissected into two tissue-sheets: inner-central sheet for transplantation and outer-peripheral sheet for qPCR to ensure retinal tissue selection. During qPCR, tissue-sheets were stored for 3-4 days using a newly developed preservation method. In a rat tumorigenicity study, no transplant-related adverse events were observed. In retinal degeneration model rats, retinal transplants differentiated into mature photoreceptors and exhibited light responses in electrophysiology assays. These results demonstrate our rationale toward self-organizing retinal sheet transplantation therapy.
\end{abstract}




\section{Introduction}

Pluripotent stem cells (PSCs) have the ability to self-organize three-dimensional (3D) neural tissue (Eiraku et al., 2011; Eiraku et al., 2008). Self-organizing aggregates with 3D-tissue are called organoids, and represent promising graft sources for cell/tissuetransplantation therapy (Clevers, 2016; Sasai, 2013; Takebe \& Wells, 2019). Toward retinal transplantation therapy, several retinal differentiation methods have been developed (Boucherie et al., 2013; Capowski et al., 2019; Eiraku et al., 2011; GonzalezCordero et al., 2017; Gonzalez-Cordero et al., 2013; Ikeda et al., 2005; Kawasaki et al., 2002; Kuwahara et al., 2015; Kuwahara et al., 2019; Lamba, Gust, \& Reh, 2009; Lamba, Karl, Ware, \& Reh, 2006; Mellough et al., 2015; Mellough, Sernagor, MorenoGimeno, Steel, \& Lako, 2012; Meyer et al., 2011; Meyer et al., 2009; Nakano et al., 2012; Osakada et al., 2008; Reichman et al., 2017; Reichman et al., 2014; Wahlin et al., 2017; Zhong et al., 2014). Among them, a self-organizing stem cell culture technology, SFEBq (serum-free floating culture of embryoid body-like aggregates with quick aggregation), is useful for 3D-retina generation (Eiraku et al., 2011; Gonzalez-Cordero et al., 2013; Kuwahara et al., 2015; Kuwahara et al., 2019; Nakano et al., 2012; Wahlin et al., 2017). We previously modified the SFEBq method by timed BMP treatment to generate 3D-retinas from feeder-free human embryonic stem cells (ESCs) and induced pluripotent stem cells (iPSCs) (Kuwahara et al., 2015; Kuwahara et al., 2019). The 3Dretinas in this culture system contain neural retinal (retinal) progenitors that expand to give rise to photoreceptors and other retinal neurons and form a multilayered stratified retinal tissue in a stage-dependent manner, recapitulating the development in vivo. 
Retinitis pigmentosa is a group of hereditary diseases characterized by progressive loss of rod photoreceptors followed by cone photoreceptors, and is the major cause of blindness in developed countries. Transplantation of photoreceptors and/or retinal progenitors is a promising therapeutic option (Gagliardi, Ben M'Barek, \& Goureau, 2019; Llonch, Carido, \& Ader, 2018; Ribeiro et al., 2021; Seiler \& Aramant, 2012). Indeed, transplantation of retinal tissues and cells from primary embryonic tissues had the potential to improve visual function (MacLaren et al., 2006; Pearson et al., 2012; Radtke et al., 2008), although the contributions of tissue/cell engraftment and donor-host material transfer remain to be studied (Pearson et al., 2016; Santos-Ferreira et al., 2016; Singh et al., 2016). To supply adequate amounts of retinal tissues and cells for cell therapy, 3D-retinas generated from PSCs represent a promising graft source. Photoreceptor precursors purified from PSC-derived retinas can survive, make contact with the host retina, and improve visual function (Gagliardi et al., 2018; GonzalezCordero et al., 2017; Gonzalez-Cordero et al., 2013; Lamba et al., 2009; Ribeiro et al., 2021; Zhu, Cifuentes, Reynolds, \& Lamba, 2017). Mouse retinal tissue sheets (retinal sheets hereafter) dissected from mouse PSC-derived 3D-retinas became engrafted into end-stage retinal degeneration model (rd1) mouse retinas and improved visual function measured by light-guided behavior tests (Assawachananont et al., 2014; Mandai et al., 2017). Human PSC-retinal sheets transplanted in immunodeficient severe retinal degeneration model mice (NOG-rd1-2J) and rats (SD-Foxn1 Tg(S334ter)3Lav nude rats; RD-nude rats hereafter), in which genetic mutations induce photoreceptor degeneration, exhibited functional maturation that resulted in restoration of light 
responses measured by ex vivo electrophysiology assays with a multi-electrode array (MEA) (Iraha et al., 2018; Tu et al., 2019). Human PSC-retinal sheets transplanted in RD-nude rats contributed to improved visual function measured by optokinetic testing and electrophysiological recording in the superior colliculus (McLelland et al., 2018). Human PSC-retinal sheets transplanted in primate models of retinal degeneration became engrafted for 2 years, differentiated into rod and cone photoreceptors to form an outer segment (OS)-like structure, and directly contacted the host bipolar cells (Shirai et al., 2016; Tu et al., 2019). Furthermore, hPSC-derived 3D-retinas had low immunogenicity and immunosuppressive properties, which may contribute to long-term engraftment (Yamasaki et al., 2021). These preclinical transplantation studies indicate that PSC-derived retinal sheets have the potential to improve visual function.

Toward clinical applications on PSC-derived retinal sheets, the establishment of a quality control (QC) strategy for 3D-retinas (intermediate products) and dissected retinal sheets (final products) has remained a major challenge. Self-organizing culture has been improved to reduce the variation in organoids at both the intra-batch and interbatch levels. Eiraku and colleagues developed the SFEBq method to regulate the size and quality of PSC-derived aggregates (Eiraku et al., 2011; Eiraku et al., 2008). We developed a modified culture system to reduce the variety of organoid quality, such as the organoid morphologies and proportions of neural retina (retina) and retinal pigment epithelium (RPE) (Kuwahara et al., 2015; Kuwahara et al., 2019), although individual organoids still exhibit diverse 3D morphologies and multiple off-target tissues. Some off-target tissues were identified as RPE tissue and cortex-like tissue (Kuwahara et al., 
2015; Meyer et al., 2011). Our previous transplantation studies used PSC lines harboring fluorescent protein knock-in reporter lines for Rx (also called Rax) and Crx to label retinal cells suitable for transplantation (Iraha et al., 2018; Nakano et al., 2012; Shirai et al., 2016). However, xenogeneic fluorescent protein knock-in reporter lines are not ideal for clinical applications. Here we establish a new QC strategy to select retinal tissue in the organoids and perform preclinical studies toward retinal sheet transplantation therapy. First, we performed gene expression analyses to identify the major off-target tissues. Next, we developed a quantitative polymerase chain reaction (qPCR)-based test by dissecting each neuroepithelium into two tissue-sheets: innercentral sheet for transplantation (named 'cap') and outer-peripheral sheet for QC test (named 'ring'). Because we found that gene expression in the ring was similar to that in the cap, the results of the QC test for the ring can be used to estimate gene expression in the cap. During this QC test (ring-PCR test hereafter), the dissected cap was stored using a newly developed preservation method. We generated retinal sheets using this ring-PCR test and preservation method and performed preclinical safety and efficacy studies to demonstrate our rationale toward use of self-organizing retinal sheets for retinal transplantation therapy. 


\section{Results}

\section{Self-organizing culture of human iPSCs for 3D-retina and dissected retinal sheet generation}

Toward retinal tissue-transplantation therapy, we developed a robust retinal differentiation method consisting of five processes based on previous studies (Assawachananont et al., 2014; Eiraku et al., 2011; Kuwahara et al., 2015; Kuwahara et al., 2019; Nakano et al., 2012; Shirai et al., 2016): 1) maintenance culture with feederfree hPSC culture and preconditioning methods, 2) retinal differentiation with SFEBq and BMP methods, 3) induction-reversal culture, 4) maturation culture, and 5) dissection (Figure 1A).

In Process 1, human iPSC cell lines were maintained on LM511-E8 matrix in StemFit medium (Miyazaki et al., 2012; Nakagawa et al., 2014). Four iPSC cell lines, iPSC-S17, iPSC-LPF11, iPSC-1231A3, and iPSC-QHJI01s04 (iPSC-Q), were stably expanded in this feeder-free maintenance culture, and their doubling time was approximately $12-18 \mathrm{~h}$. iPSCs were preconditioned with SB431542 (SB; inhibitor of TGF-beta/Nodal/Activin signaling) plus smoothened agonist (SAG; Shh signaling agonist) for 18-30 h prior to differentiation (SB+SAG hereafter) (Kuwahara et al., 2019). In Process 2, 3D-retinal differentiation culture was carried out using the SFEBqBMP method. iPSCs were dissociated into single cells, plated in V-bottom 96-well plates, and cultured in differentiation medium (growth factor-free CDM [gfCDM] + Knockout Serum Replacement [KSR]) in the presence of Y-27632 and SAG (Kuwahara et al., 2019). The resulting iPSC-aggregates were treated with BMP4 on day 3 and 
cultured in gfCDM+KSR medium (Kuwahara et al., 2015). On day 14, the iPSCaggregates were fixed and immunostained for retinal progenitor markers Pax6 and Chx10 (also called Vsx2) (Liu et al., 1994). The iPSC-aggregates were found to contain a neuroepithelium on their surface, and the majority of the cells in the neuroepithelium were positive for Chx10 and Pax6, indicating that iPSCs self-formed immature retinal tissue (Figure 1B). In Process 3, the immature retinal tissue was cultured using an induction-reversal culture method to precisely regulate the proportions of neural retina (retina) and RPE (Kuwahara et al., 2015). The immature retinal tissue was cultured with CHIR99021 (CHIR; Wnt agonist) and SU5402 (SU; FGFR inhibitor) for 3 days to bias the cells toward the RPE fate and then further cultured in retina maturation medium containing serum and taurine for 23 days to obtain an induction-reversal intermediate. In Process 4, the induction-reversal intermediate was further cultured in retina maturation medium containing serum, taurine, and T3 for 20-60 days to obtain the 3Dretina. At days 60-100, the 3D-retina was fixed and immunostained for Chx10, Pax6, Crx (photoreceptor precursor marker) (Furukawa, Morrow, \& Cepko, 1997), and Recoverin (photoreceptor marker). The 3D-retina was found to contain a multilayered retinal tissue, comprising a photoreceptor precursor layer with $\mathrm{Crx}^{+}$and $\mathrm{Recoverin}^{+}$ cells on the outer surface, a retinal progenitor layer with $\mathrm{Chx} 10^{+} / \mathrm{Pax} 6^{+}$cells in the middle, and a neuronal layer with $\mathrm{Chx} 10^{-} / \mathrm{Pax}^{++}$cells on the inner side (Figure 1B). Similar results were obtained for other iPSC lines, iPSC-LPF11 using preconditioning with SB+SAG or SAG-only and iPSC-Q (Figures 1-figure supplement 1A-C).

We further cultured 3D-retinas in long-term maturation medium containing 
all-trans retinoic acid to examine the expression of retinal maturation markers. On day 180, the 3D-retinas were fixed and immunostained. The 3D-retinas were confirmed to contain a multilayered retinal tissue, comprising a photoreceptor precursor layer with $\mathrm{Crx}^{+}$and Recoverin ${ }^{+}$on the outer surface, a retinal progenitor layer with $\mathrm{Chx} 10^{+} / \mathrm{Pax}^{+}$ cells in the middle, and a neuronal layer with $\mathrm{Chx} 10^{-} / \mathrm{Pax} 6^{++}$cells and $\mathrm{Lhx} 2^{+}$cells on the inner side (Figures $1 \mathrm{~B}$ and $\mathrm{C}$ ). $\mathrm{NRL}^{+}, \mathrm{RXRG}^{+}$, and Cone-arrestin ${ }^{+}$cells were found in the outer surface of the 3D-retina, indicating differentiation into rod precursors and cone precursors (Figure 1C). Some Recoverin ${ }^{+}$cells expressed CtBP2 (also called Ribeye), suggesting that iPSC-derived photoreceptors had the ability to express photoreceptor synaptic proteins (Figures 1C and Figure 1-figure supplement 1D).

To examine the mRNA levels of retinal and PSC marker genes during retinal differentiation culture, we performed qPCR analyses of iPSC-S17-derived cell aggregates on days $0,6,14,45,60$, and 80 (Figure 1D). On day 6 , the mRNA level of PSC marker Oct3/4 was decreased and that of anterior-neural and retinal marker Rx was increased. On day 14, the mRNA levels of Rx and Chx10 were increased. On day 45, the mRNA levels of Crx, Recoverin, and cone precursor marker RXRG were increased. Similar gene expression patterns during retinal differentiation were observed in iPSCQ-derived cell aggregates (Figure 1-figure supplement 1E), demonstrating the robustness of this self-organizing retinal differentiation culture.

In Process 5 after maturation culture, 3D-retinas at days 60-100 were dissected into retinal sheets (final products) (Figure 1E) (Assawachananont et al., 2014; Shirai et al., 2016). To check the morphology of the retinal sheets, we performed 
immunohistochemical (IHC) analysis and observed a multilayered continuous retinal tissue with a photoreceptor precursor layer containing $\mathrm{Crx}^{+}$and $\mathrm{Recoverin}{ }^{+}$cells in the outer surface and a retinal progenitor cell layer with $\mathrm{Chx} 10^{+}$and $\mathrm{Rx}^{+}$cells on the inner side (Figure 1F). Using these five processes, human allogeneic iPSC-derived retinal sheets were reproducibly generated.

\section{Characterization of major off-target tissues in the retinal differentiation culture}

The self-organizing culture mimicked embryonic development to induce both retinal tissue and off-target tissues. We sought to identify the major off-target tissues in this culture based on the morphology of the aggregates. iPSC-LPF11 cells differentiated toward the retinal fate by self-organizing culture were subjected to bright-field microscopy analysis. We found that iPSC-aggregates self-organized into retinal tissue and three off-target tissues that had different morphologies from the retinal tissue (offtarget tissue-1, -2, and -3) (Figure 2A). The typical morphology of the retinal tissue was the multilayered continuous neuroepithelium structure with a bright outer layer and a brown inner layer (Figures 1B and 2A). The typical morphologies of the off-target tissues were pigmented and/or non-pigmented thin-wrinkled epithelium (off-target tissue-1), sticky cell aggregates with a rough outer surface (off-target tissue-2), and dark-colored aggregates with a clogged inner part (off-target tissue-3) (Figure 2A). We differentiated six independent culture batches from iPSC-LPF11 and iPSC-S17 cells and examined the proportions of aggregates containing retinal tissue, off-target tissue-1, off- 
target tissue-2, and off-target tissue-3 (Figure 2B, $n=59-95$ aggregates per each batch). For all batches, most aggregates contained retinal tissue and the proportion of aggregates containing off-target tissue-1 tended to be higher than the proportion of aggregates containing off-target tissue-2 or off-target tissue-3.

Subsequently, we performed gene expression analyses to identify the cell lineages in the off-target tissues. iPSC-derived off-target tissue-1, off-target tissue-2, and off-target tissue-3 were manually dissected, possibly with small amounts of surrounding tissues, and the mRNA levels of key genes associated with fate choice, differentiation, and/or patterning were determined by qPCR analysis. Based on the $\mathrm{Ct}$ values for each gene, we calculated Z-scores for each tissue and plotted as a heat map to analyze the gene expression patterns in the tissues (Figure 2C). We found that off-target tissue-1 expressed Aqp1 and Mitf, indicating that it was eye-related tissue with RPE and ciliary body (Hamann et al., 1998; Stamer, Bok, Hu, Jaffe, \& McKay, 2003). Off-target tissue-2 expressed Emx2, indicating that it was embryonic cortex-like tissue (Theil, Aydin, Koch, Grotewold, \& Rüther, 2002). Off-target tissue-3 showed high expression of homeobox gene HoxB2 (Gavalas, Ruhrberg, Livet, Henderson, \& Krumlauf, 2003; Zhai, Lin, Canete-Soler, \& Schlaepfer, 2005). Undifferentiated hPSC marker Lin28a was not detected in the three off-target tissues or retinal tissue (Kuroda et al., 2012). We performed IHC analyses and confirmed that off-target tissue-1 was positive for RPE and ciliary body marker Aqp1, while off-target tissue-2 was positive for embryonic cortical (dorsal telencephalic) marker Emx2 (Figure 2D). Similar results were obtained for iPSC-S17 cells (Figure 2 - figure supplement 1A). To investigate the 
gene expression in off-target tissue-3 more deeply, we performed a microarray analysis and found that it expressed multiple Hox genes, including HoxA1, HoxA3, HoxA4, HoxA5, HoxB2, HoxB3, HoxB4, HoxB5, HoxB6, HoxB7, HoxB8, and HoxB9, indicating that it was posterior-neural spinal cord-like tissue (Figure 2E).

We further investigated the gene expressions in the retinal tissues and offtarget tissues derived from iPSC-LPF11, iPSC-S17, and iPSC-Q cells. Principal component analysis (PCA) showed that the gene expression patterns in all retinal tissues were quite similar and that the off-target tissues differed from the retinal tissues (Figure 2 -figure supplement 1B).

Collectively, using this self-organizing culture method, hPSCs robustly differentiated to form a multilayered retinal tissue and the major off-target tissues were $\mathrm{Aqp}^{+}$eye-related tissue (off-target tissue-1), Emx $2^{+}$cortex-like tissue (off-target tissue2), and HoxB2 ${ }^{+}$spinal cord-like tissue (off-target tissue-3).

\section{QC method for retinal sheets by testing gene expression in the surrounding outer tissue-sheet}

The self-organized retinal tissue had a unique multilayered continuous neuroepithelium structure (Figures 1B, 1C, 2A, and Figure 1-figure supplement 1A). Therefore, we can identify the retinal tissue by carefully observing the aggregates under a bright-field microscope during the dissection process (Process 5). However, PSCs in this culture system differentiated into not only retinal tissue but also off-target tissues (Figure 2), 
providing a potential risk that off-target tissues will be dissected to generate the final product. Toward clinical applications, we aimed to develop a fail-safe QC method to ensure the selection of transplantable retinal sheets and avoid including off-target tissues by mistake. Although the qPCR-based QC test for all retinal sheets is a very sensitive way to detect off-target tissues, execution of this test leads to the destruction of all transplantable retinal sheets. To avoid this destruction, we decided to dissect the continuous neuroepithelium into two tissue-sheets, comprising the inner-central sheet for transplantation (cap) and the other outer-peripheral sheet for QC test (ring) (Figure 3A), and to carry out the QC test for each retinal sheet by analyzing the gene expression in the ring.

iPSC-LPF11 cells were differentiated into the retinal fate by self-organizing culture (Figure 1A) and the continuous neuroepithelium produced was dissected into the inner-central sheet (cap) and the outer-peripheral sheet (ring) (Figure 3A). On IHC analysis, the cap and ring were both found to contain a multilayered continuous retinal tissue comprising a photoreceptor precursor layer with $\mathrm{Crx}^{+}$and $\mathrm{Recoverin}^{+}$cells on the outer surface and a retinal progenitor cell layer with $\mathrm{Chx} 10^{+}$and $\mathrm{Pax} 6^{+}$cells on the inner side (Figure 3B).

We compared the gene expression patterns in the caps and rings. Various neuroepithelia were dissected into cap and ring tissues, and the RNA was extracted. We performed qPCR analysis, calculated the Z-scores from the $\mathrm{Ct}$ values for each cap and ring, and plotted the scores as a heat map to visualize the gene expression (Figure 3C). We found that the gene expression patterns in each cap and ring from the same 
neuroepithelium were quite similar. To statistically analyze the similarity between the caps and the rings, we drew regression lines for the gene expression in each cap and ring from the same neuroepithelium and calculated the coefficient of determination $\left(\mathrm{R}^{2}\right)$. We found that the $\mathrm{R}^{2}$ values for all sets of cap and ring were almost 1.0 (Figure 3D). Similar results were obtained using iPSC-S17 cells (Figures 3-figure supplement $1 \mathrm{~A}$ and $\mathrm{B})$.

The above results demonstrated that the gene expression patterns of retinal and off-target markers in the cap and ring were similar. Therefore, we decided to perform the qPCR-based QC test using the ring to estimate the gene expression in the cap (ring-PCR test hereafter). By performing the ring-PCR test, we can evaluate whether the selected cap contains retinal tissue, does not contain large amount of offtarget tissues, and is applicable for transplantation.

\section{Development of a controlled room temperature non-freezing preservation method}

Between the dissection process and retinal sheet transplantation, it is necessary to perform QC tests including the ring-PCR test to select transplantable retinal sheets. However, it requires at least 1-2 days to complete the ring-PCR test. Therefore, we aimed to develop a preservation method for retinal tissue. Although retinal tissue can be frozen and thawed as reported previously (Nakano et al., 2012; Reichman et al., 2017), subretinal transplantation of frozen retinal tissue in nude rats showed impaired engraftment (data not shown). We therefore developed a non-freezing preservation 
method.

We first examined the optimal medium and temperature for non-freezing preservation of iPSC-derived 3D-retinas and dissected retinal sheets. To determine the optimal medium, we evaluated retinal maturation medium, University of Wisconsin (UW) solution, balanced salt solution (BSS), and Optisol-GS (Optisol hereafter), as candidate preservation solutions (Ishikawa et al., 2015; Means et al., 1996; Nahar et al., 2018), at 4,17 , and $37^{\circ} \mathrm{C}$. iPSC-derived 3D-retinas were generated, preserved for 3 days under various conditions, and cultured in retinal maturation medium at $37^{\circ} \mathrm{C}$ for 7 days as recovery culture (Figures $4 \mathrm{~A}$ and $\mathrm{B}$ ). The preserved retinas and control retinas were fixed and immunostained to check the morphology of the retinal tissue. We found that preservation at $17^{\circ} \mathrm{C}$ with BSS or Optisol were optimal conditions to maintain the multilayered continuous retinal epithelium structure (Figure 4A). Preservation at $4{ }^{\circ} \mathrm{C}$ was not a preferred condition, possibly causing damage to the retina. UW solution, a golden standard for preserving peripheral tissues including the pancreas, was also not preferred. One of the differences between UW solution and Optisol was the concentration of potassium chloride $(\mathrm{KCl}): 120 \mathrm{mM}$ in UW solution and $5.33 \mathrm{mM}$ in Optisol. Indeed, Optisol supplemented with $\mathrm{KCl}$ at $120 \mathrm{mM}$ impaired the morphology of the retinal tissue (Figure 4-figure supplement 1A). Consistently, the concentration of $\mathrm{KCl}$ in $\mathrm{BSS}$ and DMEM was $5.3 \mathrm{mM}$ and preservation at $17^{\circ} \mathrm{C}$ in $\mathrm{BSS}$ and DMEM was found to be preferable (Figures 4A and Figure 4-figure supplement 1B). These results raise the possibility that the concentration of $\mathrm{KCl}$ might be a key factor for the preservation solution. Based on these results, we chose Optisol for the non-freezing 
preservation solution, because Optisol is widely clinically used for human corneal transplantation (Means et al., 1996).

To determine the optimal temperature for preservation, 3D-retinas were preserved in Optisol at $4,12,15,17,20,22$, and $37^{\circ} \mathrm{C}$ for 3 days and then cultured in retinal maturation medium at $37^{\circ} \mathrm{C}$ for 7 days as the recovery culture (Figure 4B). The preserved retinas and control retinas were fixed and immunostained for a photoreceptor precursor marker (Crx) and a nuclear marker (DAPI). The numbers of $\mathrm{Crx}^{+}$cells and nuclei were counted in the continuous neuroepithelium and the proportions of $\mathrm{Crx}^{+}$cells were calculated. The proportions of $\mathrm{Crx}^{+}$cells were higher at $12,15,17,20$, and $22{ }^{\circ} \mathrm{C}$ than at 4 and $37^{\circ} \mathrm{C}$ (Figure 4C). Notably, the proportions of $\mathrm{Crx}^{+}$cells at 17, 20, and $22{ }^{\circ} \mathrm{C}$ were similar to the proportions of $\mathrm{Crx}^{+}$cells in the control culture. These results suggest that preferable range of preservation temperature was $17 \pm 5^{\circ} \mathrm{C}$ and the optimal temperature was $17-22^{\circ} \mathrm{C}$.

We examined the cell viability and gene expression in retinas preserved in Optisol at $17^{\circ} \mathrm{C}$ for 4 days. The cell viability in the preserved retinas exceeded $95 \%$ and was similar to that in control retinas in culture (Figure 4D). The mRNA levels of Chx10 and $\mathrm{Crx}$ in the preserved retinas were comparable to those in control retinas in culture (Figure 4E). Furthermore, the mRNA levels of Chx 10 and Crx in retinas preserved in Optisol at $17{ }^{\circ} \mathrm{C}$ for 7 days were comparable to those in control retinas in culture (data not shown). Collectively, these results demonstrated that non-freezing preservation in Optisol at $17^{\circ} \mathrm{C}$ was the optimal condition for retinal tissue.

We analyzed the status of retinal tissues preserved in Optisol at $17^{\circ} \mathrm{C}$ by IHC 
and proliferation analyses. Control retinas before preservation $(\mathrm{d} 70+0)$, retinas preserved in Optisol at $17^{\circ} \mathrm{C}$ for 2 days $(\mathrm{d} 70+2)$ and 4 days $(\mathrm{d} 70+4)$, and retinas recovered by culture at $37^{\circ} \mathrm{C}$ for 3 days $(\mathrm{d} 70+4+3)$ and 7 days $(\mathrm{d} 70+4+7)$ were treated with the thymidine analog 5-ethynyl-2'-deoxyuridine (EdU) for $4 \mathrm{~h}$ to label proliferating cells in S-phase of the cell cycle. The retinas were fixed, and the incorporated EdU was stained with azide labeled with fluorescent dye in combination with IHC staining. Expression of Ki67 was detected in both control retinas in culture and retinas preserved at $17{ }^{\circ} \mathrm{C}$ (Figure $4 \mathrm{~F}, \mathrm{~d} 70+2$ and $\left.\mathrm{d} 70+4\right)$. In contrast, $\mathrm{EdU}^{+}$cells were not detected in retinas preserved at $17^{\circ} \mathrm{C}$ (Figures 4F, 4G, and Figure 4-figure supplement 1C, d70+2 and $\mathrm{d} 70+4)$, indicating that preserved retinal progenitors were arrested before entering S-phase. Importantly, EdU uptake was upregulated during recovery culture, indicating that the proliferation ability was reversible (Figures 4F and G). Similar results for EdU uptake were observed in retinas preserved at $17^{\circ} \mathrm{C}$ in another preservation medium (Figure 4-figure supplement 1D). The number of cleaved caspase- $3^{+}$cells (apoptotic cells) was slightly high in the preserved retinas, while the numbers in the recovered retinas $(d 70+4+3$ and $d 70+4+7)$ were similar to that in control retinas, suggesting that the increase in apoptosis or non-apoptotic caspase- 3 cleavage during preservation may be transient (Figure 4-figure supplement 1C).

By combining the new controlled room temperature non-freezing preservation method (RT-preservation method hereafter) with the ring-PCR test, we developed a QC method for the dissection of 3D-retinas (Process 5) as follows: 1) dissection of the cap and ring from each $3 \mathrm{D}$-retina, 2) preservation of the caps at $17^{\circ} \mathrm{C}$ by the RT- 
preservation method until release, 3) qPCR analysis to select passed and failed rings, 4) exclusion of caps corresponding to failed rings to obtain the retinal sheets as the final products, and 5) shipping of the retinal sheets for transplantation (Figure 4H).

\section{In vivo tumorigenicity study by subretinal transplantation in nude rats}

We developed methods to generate retinal sheets from iPSC-S17, iPSC-LPF11, and iPSC-Q cells (Figures 1-4). Self-organized 3D-retinas from the three iPSC lines formed similar multilayered retinal tissues comprising a photoreceptor precursor layer and a retinal progenitor cell layer (Figures 1 and Figure 1 -figure supplement 1 ). The gene expression patterns in the dissected retinal sheets from the three iPSC lines showed consistent quality (Figures 2, 3, Figure 2-figure supplement 1, and Figure 3-figure supplement 1). We then used the developed ring-PCR test and RT-preservation method to obtain transplantable retinal sheets (Figures 3 and 4). By using these methods, we generated the retinal sheets and performed a tumorigenicity study as an in vivo safety study.

An in vivo tumorigenicity study was conducted by subretinal transplantation in immunodeficient nude rats. Retinal sheets were generated from iPSC-Q cells and stored by the RT-preservation method for 3-4 days. The rats were divided into intact, sham surgery, and transplanted groups, and the rats in the latter group underwent transplantation of one retinal sheet in the subretinal space (Figure 5-figure supplement 1A, intact $[n=8]$, sham $[n=12]$, transplanted $[n=21])$. The each transplanted retinal sheet (graft) was properly located in the subretinal space as confirmed by fundus 
imaging and optical coherence tomography (OCT) analysis (Figure 5-figure supplement 1B).

The transplanted rats showed no abnormalities in their body weights, survival rates, and clinical signs compared with intact or sham-operated rats during the observation period of up to 78 weeks after transplantation (Figures 5A and B).

Histopathological analysis of the grafts at 13, 26, 52, and 78 weeks after transplantation revealed no proliferative or malignant features such as nuclear atypia (Figures 5C and D). IHC analyses of grafts were then conducted (Figures 5E-I). The percentages of proliferating human cells $\left(\mathrm{Ki}_{6} 7^{+}\right.$and $\left.\mathrm{Ku} 80^{+}\right)$among the human cells $\left(\mathrm{Ku} 80^{+}\right)$were $<1 \%$ at each time point, indicating very limited proliferation of the graft cells (Figures 5F, H, and I) (Kanemura et al., 2014; Sugai et al., 2021). IHC staining for the retinal cell markers and human nuclear marker $\mathrm{HuNu}$ showed differentiation of the graft cells into photoreceptors (Recoverin ${ }^{+}$and $\left.\mathrm{HuNu}^{+}\right)$and bipolar cells $\left(\mathrm{Chx} 10^{+}\right.$and $\mathrm{HuNu}^{+}$) (Figure 5E). Specifically, the iPSC-Q-derived retinal sheets became engrafted as retinal tissue including photoreceptors at $>1.5$ years after transplantation even under the xenotransplantation condition. Interestingly, some human retinal grafts, possibly transplanted in not only the subretinal space but also the vitreous cavity, became engrafted and survived in the vitreous cavity and differentiated into photoreceptors with mature morphology, indicating that the retinal sheets had the potential to generate photoreceptors with the mature morphology (Figures 5G and Figure 5-figure supplement 1C).

In this study, no tumorigenicity and no other adverse effects related to the iPSC-Q-derived retinal sheets were observed. 


\section{Preclinical efficacy study by subretinal transplantation in retinal degeneration model rats}

To examine whether retinal sheets stored by the RT-preservation method for $3-4$ days could become engrafted and differentiate into mature photoreceptors in severely degenerated condition, we performed a transplantation study using end-stage retinal degeneration model nude rats (RD-nude rats) carrying a mutated human rhodopsin transgene (SD-Foxn1 Tg(S334ter)3-LavRrrc line) (Sagdullaev, Aramant, Seiler, Woch, \& McCall, 2003; Seiler et al., 2014). iPSC-S17-derived retinal sheets stored by the RTpreservation method for 3-4 days were subretinally transplanted in RD-nude rats aged 20-30 weeks. At 24-44 weeks after transplantation, the eyecups were fixed and subjected to IHC analysis. In the RD-nude rats, the host/rat photoreceptors were almost completely degenerated at this stage (Figure 6A, control area). We found that human photoreceptors double-positive for human Ku80 and Recoverin became engrafted in the severely degenerated rat retinas to form photoreceptor rosette structures (Figure 6A'), consistent with previous studies (Iraha et al., 2018; Kuwahara et al., 2019; Shirai et al., 2016; Tu et al., 2019). In addition, iPSC-Q-derived retinal sheets stored by the RTpreservation method for 2-3 days were transplanted in the subretinal space of RD-nude rats. We found that these iPSC-Q-derived retinal sheets also became engrafted in degenerated rat retinas and that human photoreceptors double-positive for $\mathrm{HuNu}$ and Recoverin formed photoreceptor rosette structures (Figures 6-figure supplement 1A- 
C). These results demonstrated that iPSC-derived retinal sheets preserved at $17^{\circ} \mathrm{C}$ for 2-4 days had the potential to become engrafted and differentiate into photoreceptors in vivo.

We examined whether preserved iPSC-retinal sheets transplanted in the severely degenerated retina could differentiate into mature photoreceptors with the OSlike structure. The photoreceptor rosettes contained both cone photoreceptor marker Cone-arrestin-positive cells and rod photoreceptor marker S-arrestin-positive cells (Figures 6B, B', and B'). The photoreceptor rosettes contained $\mathrm{NRL}^{+}$rod precursors, $\mathrm{RXRG}^{+}$cone precursors, and some mature cone photoreceptors positive for S-opsin and L/M-opsin (Figures 6C, C', and D). Importantly, the photoreceptor rosettes contained rod photoreceptors double-positive for rod phototransduction marker GNAT1 and OSmarker PRPH2 and cone photoreceptors double-positive for cone phototransduction marker GNAT2 and OS-marker PNA (Figures 6E, E', and F). These results demonstrated that preserved iPSC-retinal sheets became engrafted into severe RDmodel immunodeficient rats and differentiated into mature rod and cone photoreceptors with OS-like structures.

To demonstrate the maturation of iPSC-derived photoreceptors, we examined the outer plexiform layer (OPL) structure and the expressions of photoreceptor-synapse markers. The thickness of the human photoreceptor layer in rosettes was $2-5$ cells (Figure 6B'). We observed that Synaptophysin, a major synaptic vesicle protein, positive neurites and Calbindin-positive horizontal cells resided around the human photoreceptor rosettes, indicating that an OPL-like structure was formed (Figures 6G, 
G', G”, H, and H', arrowheads). Examination of the human photoreceptors at higher magnification revealed that Recoverin ${ }^{+}$and $\mathrm{Ku} 80^{+}$human photoreceptors were in direct contact with PKCalpha ${ }^{+}$and $\mathrm{Ku} 80^{-}$host/rat bipolar cells (Figures 6I and I', arrowheads). We also observed expression of the photoreceptor pre-synaptic markers CtBP2 (Ribeye) and LRIT3 at the tips of PKCalpha ${ }^{+}$bipolar dendrites (Figures 6J and J'). These results demonstrated that preserved iPSC-retinal sheets differentiated and matured to form human photoreceptor rosettes with an OPL-like structure and expression of photoreceptor-synapse markers.

To demonstrate the functional potential of the retinal sheets, we performed ex vivo electrophysiology assays with an MEA to examine the light responsiveness of iPSC-retinal sheets after transplantation. iPSC-S17-derived retinal sheets on differentiation days 81-95 after RT-preservation for 3 days were transplanted in the subretinal space of 12 RD-nude rats aged 20-30 weeks. Non-transplanted eyes of RDnude rats were used as age-matched controls. The rats were subjected to MEA recordings at approximately 60 weeks of age, i.e., $8-9$ months after transplantation, when almost no retinal ganglion cell (RGC) light responses attributed to residual host/rat photoreceptors were observed for scotopic-to-mesopic light stimuli (10.56 log photons $/ \mathrm{cm}^{2} / \mathrm{s}$ ) (Tu et al., 2019). Isolated retinas from the transplanted and nontransplanted rats were separately mounted on the MEA electrodes (Figures 7A and B). The light stimuli-induced electrophysiological patterns were analyzed to sort out the RGC-derived spikes (RGC-spikes), and the RGC-spike counts were determined to evaluate the RGC light responses as described previously (Matsuyama et al., 2021; Tu 
et al., 2019). Light stimuli at three different intensities (weak: $10.56 \log$ photons $/ \mathrm{cm}^{2} / \mathrm{s}$; medium: $12.16 \log$ photons $/ \mathrm{cm}^{2} / \mathrm{s}$; strong: $12.84 \log$ photons $/ \mathrm{cm}^{2} / \mathrm{s}$ ) were applied to the transplanted and non-transplanted retinas. The electrophysiological recordings were performed under three conditions: before addition of metabotropic glutamate receptor (mGluR6) blocker L-AP4 (Before), after addition of L-AP4 (L-AP4), and after washout of L-AP4 (After washout) (Figure 7A). The peri-stimulus time histograms of the RGCspike counts suggested that light stimulus-induced RGC-spikes were detected in the transplanted retina (Figure 7C, upper). In a representative recording (Figure 7C, box and Figure 7D), increased RGC-spike counts were observed after light stimulus onset (Figure 7D, Before). L-AP4 addition attenuated the light stimulus-induced RGC-spikes (Figures 7C and D, L-AP4). After washout of L-AP4, light stimulus-induced RGCspikes reappeared with enhanced responsiveness (Figure 7D, After washout). In contrast, few light stimulus-induced RGC-spikes were detected in the non-transplanted control retinas (Figures 7C and D, bottom). These results indicated that the RGC light responses in the transplanted rat retinas were derived from the grafted human retinal sheets and were dependent on synaptic transmission from grafted photoreceptors to bipolar cells. Similar RGC light responses were evident in 2 of 13 eyes transplanted with iPSC-Q-derived retinal sheets after RT-preservation (Figures 7-figure supplement 1A and B). Even iPSC-retinal sheets transplanted after RT-preservation for 4 days showed RGC light responses (Figure 7-figure supplement $1 \mathrm{C}$ and D).

To further confirm the preclinical efficacy of the retinal sheets, we compared the probability of RGC light responses in non-transplanted (Control) and transplanted 
retinas (Figure 7E). The RGC light response probability was estimated by statistical modeling using Bayesian statistical inference as previously described (Matsuyama et al., 2021). In non-transplanted rat retinas, robust RGC light responses were rarely detected for the weak, medium, and strong light stimuli (Figure 7E, Control and Before; 0 light responses in 12 rat retinas, $0 \%$ ). In rat retinas transplanted with iPSC-S17derived retinal sheets, robust RGC light responses with average response probability $>$ 0.25 were observed in two rat retinas for medium-to-strong light stimuli (Figure 7E, Transplanted and Before; 2 light responses in 12 rat retinas, 17\%). The detected RGC light responses were dominantly ON type because they showed attenuation in the presence of L-AP4 (Figure 7E, Transplanted and L-AP4) and reappeared after L-AP4 was washed out (Figure 7E, Transplanted and After washout). Note that RGC lightresponses were enhanced after L-AP4 washout (Figure 7E, Transplanted and After washout versus Transplanted and Before), consistent with the previous study (Matsuyama et al., 2021). After L-AP4 washout, RGC light responses were observed in three transplanted retinas for weak light stimuli and six transplanted retinas for mediumto-strong light stimuli (Figure 7E, Transplanted and After washout; 6 light responses in 12 rat retinas, 50\%). These results demonstrated that iPSC-retinal sheets transplanted after RT-preservation exhibited RGC light response functions (6 light responses in 12 transplanted rat retinas [50\%] versus 0 light responses in 12 control rat retinas $[0 \%]$ ). Interestingly, RGC light responses were observed in retinal sheets transplanted on differentiation days 81-83 $(n=7)$ and 90-95 $(n=5)$, indicating that retinal sheets at approximately day 80 and approximately day 90 both had the potential 
to improve light responses in retinal degeneration model rats and that the allowable range of differentiation days for the efficacy may be wide (Figure 7-figure supplement 2).

Taken together, the efficacy studies demonstrated that iPSC-derived retinal sheets preserved at $17^{\circ} \mathrm{C}$ for 3-4 days had the potential to become engrafted and mature in the severely degenerated RD-nude rat retinas to exhibit the RGC light response functions measured by ex vivo electrophysiology assays. These safety and efficacy studies on the retinal sheets demonstrated that the QC and RT-preservation methods were reasonable for the generation of transplantable retinal sheets. 


\section{Discussion}

Self-organizing stem cell culture technology enables the generation of 3D-tissues and organoids in a dish (Eiraku et al., 2011; Eiraku et al., 2008; Meyer et al., 2011; Sasai, 2013). Sasai and colleagues and our group previously developed a robust retinal differentiation method based on 3D-self-organizing culture (Eiraku et al., 2011; Kuwahara et al., 2015; Kuwahara et al., 2019; Nakano et al., 2012). Tissue transplantation is an attractive approach for regenerative medicine in retinal degeneration, because retinal tissue can become engrafted to generate photoreceptors with an OS-like structure and an OPL-like structure even in severely degenerated retina. Indeed, hPSC-retinal sheets were engrafted for $0.5-2$ years in photoreceptor degeneration model rodents and non-human primates in previous studies (Iraha et al., 2018; Kuwahara et al., 2019; Lin et al., 2020; McLelland et al., 2018; Shirai et al., 2016; Tu et al., 2019) and for 1.5 years in nude rats in the present study. The key remaining technologies for tissue/organoid transplantation were a QC strategy and a preservation method. In this study, we developed a QC strategy for hPSC-derived retinal sheets. Retinal tissue in culture self-forms a unique morphological structure that is easy to distinguish under a microscope, and therefore retinal sheets can be dissected. To exclude possible errors in the selection of retinal tissue and avoidance of off-target tissues, we characterized the major off-target tissues and developed the ring-PCR test. Retinal tissue with its large continuous neuroepithelial structure can be dissected into two tissue-sheets: the inner-central sheet (cap) and the outer-peripheral sheet (ring) with similar gene expression patterns. Thus, qPCR analysis of the ring can be used to 
estimate the gene expression in the corresponding cap. As the ring-PCR test requires 12 days to complete, we developed the RT-preservation method to store retinal sheets for at least 3-4 days. We found that the temperature was important and that a controlled room temperature of approximately $17{ }^{\circ} \mathrm{C}$ was better than $4{ }^{\circ} \mathrm{C}$. To demonstrate the rationale for the QC strategy and the RT-preservation method, we performed in vivo tumorigenicity and preclinical efficacy studies. In the tumorigenicity study, no tumors or no other adverse effects related to the iPSC-derived retinal sheets were observed. The preclinical efficacy study demonstrated that preserved retinal sheets had the potential to become engrafted and mature in the severely degenerated condition and exhibited light response functions. These preclinical studies support the concept of iPSC-derived retinal-sheet transplantation for retinal degeneration.

Based on present and previous studies (Assawachananont et al., 2014; Eiraku et al., 2011; Iraha et al., 2018; Kuwahara et al., 2015; Kuwahara et al., 2019; Mandai et al., 2017; McLelland et al., 2018; Nakano et al., 2012; Radtke et al., 2008; Shirai et al., 2016; Tu et al., 2019; Yamasaki et al., 2021), Kobe City Eye Hospital applied for clinical research for a limited number of patients using iPSC-derived retinal sheets for retinitis pigmentosa. In June 2020, the clinical research plan was approved by the Health Science Council at the Ministry of Health, Labour and Welfare of Japan, and the clinical research was started (Trial ID: jRCTa050200027). Sumitomo Dainippon Pharma developed a clinical grade manufacturing process and generated clinical grade allogeneic iPSC-derived retinal sheets. iPSC-retinal sheets were transplanted in two patients in 2020-2021. 
The present study is based on a highly efficient and robust self-organizing culture technology. The combination of SFEBq, BMP, preconditioning, and inductionreversal methods allows robust self-organizing 3D-differentiation culture for retinal tissue generation with a large continuous neuroepithelium (Kuwahara et al., 2015; Kuwahara et al., 2019). This large continuous neuroepithelium structure had a size of 1$3 \mathrm{~mm}$ in the tangential direction and was easy to distinguish under the microscope, making it possible to dissect the cap and the ring. The self-organized retinal tissue with continuous neuroepithelium reproducibly differentiated to form a multilayered structure with $\mathrm{Crx}^{+}$photoreceptor precursor and $\mathrm{Chx} 10^{+}$retinal progenitor cell layers from multiple hPSC lines using our protocols or different culture protocols (Capowski et al., 2019; Gonzalez-Cordero et al., 2017; Mellough et al., 2015; Meyer et al., 2011; Nakano et al., 2012; Reichman et al., 2014; Zhong et al., 2014). This feature of 3D selforganization possibly based on the intrinsic embryonic development program is the core technology for robust generation of retinal sheets with controlled quality.

One of the features of the QC method in the present study was the dissection and analysis of the ring. By dissecting the cap and the ring from the same continuous neuroepithelium, we were able to check the quality of the cap by analysis of the ring. This cap-and-ring approach could be utilized for other organoids with a continuous epithelium structure such as cortical, hippocampal, pituitary, and intestinal organoids (Eiraku et al., 2008; Sakaguchi et al., 2015; Sato et al., 2009; Suga et al., 2011). Although manual dissection of tiny tissues is a prevalent experimental procedure in research using Xenopus early embryos or mouse developing brains for primary neural 
precursor cell culture (Assawachananont et al., 2014; Shirai et al., 2016), automation and mechanization of the dissection process will be important for future manufacture of iPSC-derived retinal sheets.

To examine the quality of the ring, one candidate QC test was qPCR analysis and the others were IHC analysis, flow cytometry, gene chip analysis (microarray), RNA-seq, and single cell analysis. We chose qPCR in this study, because it allows highthroughput assays to test many samples in a relatively short period, has high sensitivity, and is technically easy (Kuroda et al., 2012). The other QC tests also had strong points. Recent study using RNA-seq approach revealed that Six6-negative off-target cells in retinal differentiation culture using Matrigel method contain multiple neural cells including Emx2-positive cells and HoxB2-positive cells (Wahlin et al., 2021). In addition, single cell analyses such as sc-RNA-seq are powerful technologies, and several groups have reported pioneered studies (Capowski et al., 2019; Collin et al., 2019; Cowan et al., 2020; Phillips et al., 2018; Sridhar et al., 2020). Meanwhile, imaging technologies and computational image processing, such as deep-learning technologies, are a promising approach for the development of non-destructive QC tests. In future studies, we would like to proceed with single cell analysis and imaging technology to further investigate the quality of the self-organizing 3D-retinas.

Although cryopreservation is an attractive approach for worldwide supply, cryopreserved retinal sheets exhibited impaired engraftment (data not shown). Therefore, we needed to develop a non-freezing preservation method and found that the optimal temperature for retinal sheet preservation was nearly room temperature 
$\left(17 \pm 5^{\circ} \mathrm{C}\right)$. Room temperature preservation was reported for hepatic tissue (Ishikawa et al., 2015), and our study demonstrated that near room temperature was suitable for neural tissues like the retina. Our new RT-preservation method enables storage of retinal sheets for at least 3-4 days. Low temperatures around $4{ }^{\circ} \mathrm{C}$ caused damage to the tissues, possibly through abnormal intracellular metabolism at $4{ }^{\circ} \mathrm{C}$ (Ishikawa et al., 2015). Culture at $37^{\circ} \mathrm{C}$ under $5 \% \mathrm{CO}_{2}$ was suitable for cell growth, but the morphology of the retinal sheets changed during self-organizing culture. RT-preservation at $17 \pm 5^{\circ} \mathrm{C}$ could maintain the morphology of the retinal sheets. During preservation, the retinal tissue arrested to enter S-phase, raising the possibility that proliferation may become paused under this preservation condition. RT-preservation was recently reported for RPE and retinal tissue (Georgiou et al., 2020; Kitahata et al., 2019). We developed an RT-preservation method and performed transplantation studies, including a 1.5-year tumorigenicity study, for retinal sheets under the RT-preservation condition.

Toward clinical applications, we evaluated the in vivo tumorigenicity and efficacy of iPSC-retinal sheets generated using the ring-PCR test and RT-preservation method. iPSC-Q-derived retinal sheets preserved at $17^{\circ} \mathrm{C}$ became engrafted for $>1.5$ years after transplantation even under xenotransplantation conditions with no sign of tumorigenicity. We also provided evidence that RT-preserved iPSC-retinal sheets became engrafted and matured in RD-nude rats to form photoreceptor rosettes. Similar human photoreceptor rosettes were observed in previous transplantation studies using non-preserved fresh retinal sheets (Iraha et al., 2018; Kuwahara et al., 2019; Shirai et al., 2016; Tu et al., 2019). Engrafted iPSC-photoreceptors in vivo matured to form the 
OS-like structure on the inner-apical surface of the rosettes and the OPL-like structure with photoreceptor synaptic marker expression in the outer basal side adjacent to bipolar cells and horizontal cells. In future studies, electron microscopy will be a key to examining the synaptic connections between grafted photoreceptors and host bipolar cells. Importantly, 50\% of iPSC-retinal transplants exhibited light responses monitored by ex vivo MEA recordings. This rate was comparable to previous results for transplantation of fresh iPSC-retinas without RT-preservation (4 light responses in 7 transplanted rat eyes, 57\%) (Tu et al., 2019). These observations demonstrated that RTpreserved iPSC-retinal sheets transplanted in the severe retinal degeneration condition became engrafted and matured to form photoreceptor rosettes with mature photoreceptor morphology and exhibited RGC light responses.

Toward future clinical applications in large numbers of patients, surgical procedures, transplantation devices, and immunosuppression protocols are important to enable widespread use of retinal sheets. For clinical trials, further preclinical studies are necessary. Based on the self-organizing stem cell culture technology, the realization of tissue/organoid therapy in medicine is ongoing. 


\section{Materials and Methods}

\section{Human iPSC lines}

Two human iPSC lines, iPSC-LPF11 and iPSC-S17, were established from peripheral blood cells using Sendai virus vectors by Sumitomo Dainippon Pharma (Kuwahara et al., 2019). The iPSC-QHJI01s04 line (iPSC-Q) was established by the iPSC Stock Project organized by the Kyoto University Center for iPS Cell Research and Application (CiRA), and provided from Kyoto University (Doi et al., 2020). The iPSC-1231A3 line established at Kyoto University and derived from ePBMC(R) purchased from Cellular Technology Limited (http://www.immunospot.com/) was provided by Kyoto University (Nakagawa et al., 2014). The experimental protocols using clinical grade human iPSCs were approved by the Research Ethics Committee of Sumitomo Dainippon Pharma Co., Ltd., Japan.

\section{Self-organizing culture of human iPSCs for 3D-retina generation}

The feeder-free hPSC-culture, SFEBq, preconditioning, d0-SAG, BMP, and inductionreversal culture, methods were performed as described (Kuwahara et al., 2015;

Kuwahara et al., 2019; Nakagawa et al., 2014; Nakano et al., 2012) with slight modifications. In Process 1 (maintenance culture), human iPSCs were maintained on LM511-E8 matrix (Nippi) in StemFit medium (Ajinomoto) according to a published protocol (Nakagawa et al., 2014) with slight modifications (Kuwahara et al., 2019). The medium was changed every $1-2$ days until the cells reached $70 \%-80 \%$ confluence. iPSCs were passaged using TrypLE Select Enzyme (Thermo Fisher Scientific) and 
dissociated into single cells by gentle pipetting. The dissociated iPSCs were seeded at a density of 700-1700 cells/ $\mathrm{cm}^{2}$ and cultured in LM511-E8 matrix-coated six-well culture plates (Iwaki) containing StemFit medium with $10 \mu \mathrm{M}$ Y-27632 (Wako) (Kuwahara et al., 2019).Prior to differentiation, iPSCs were treated with SB431542 (SB; Wako) and/or smoothened agonist (SAG; Enzo Biochem) as the preconditioning step.

In Process 2 (retinal differentiation), hPSCs were treated with TrypLE Select Enzyme at $37^{\circ} \mathrm{C}$ for $4-7 \mathrm{~min}$, and dissociated into single cells by gentle pipetting. The dissociated iPSCs were quickly reaggregated using low-cell-adhesion 96-well plates with V-bottomed wells (Sumilon PrimeSurface plates; Sumitomo Bakelite) in differentiation medium (gfCDM+KSR) with Y-27632 and SAG (d0-SAG method). The differentiation medium was gfCDM supplemented with $10 \% \mathrm{KSR}$, while gfCDM alone comprised 45\% Iscove's modified Dulbecco's medium (Gibco), 45\% Hams F12 (Gibco), Glutamax, 1\% Chemically Defined Lipid Concentrate (Gibco), and $450 \mu \mathrm{M}$ monothioglycerol (Sigma-Aldrich). The day of SFEBq culture initiation was defined as day 0. On day 3, recombinant human BMP4 (R\&D Systems) was added at $1.5 \mathrm{nM}$ (55 ng/ml) (Kuwahara et al., 2015). Thereafter, the culture medium was changed every 3-4 days to generate immature retinal tissue.

In Process 3 (induction-reversal culture), the immature retinal tissue generated from iPSCs was subjected to a two-step induction-reversal culture as follows. For the induction culture, cell aggregates on days 14-18 were transferred from 96-well plates to 90-mm non-cell-adhesive petri dishes (Sumitomo Bakelite; approximately 32-48 aggregates/90-mm dish), and cultured for 3 days in DMEM/F12-Glutamax medium 
(Gibco) containing 1\% N2 supplement (Gibco), $3 \mu \mathrm{M}$ CHIR99021 (GSK3 inhibitor; Wako), and $5 \mu \mathrm{M}$ SU5402 (FGFR inhibitor; Wako). For the reversal culture (Process 3) and the maturation culture (Process 4), the cell aggregates were cultured in retina maturation medium as described (Nukaya et al. in preparation; WO2019017492A1, WO2019054514A1). The medium was changed every third or fourth day to obtain 3Dretinas. Floating cell aggregates were analyzed using an inverted microscope (Keyence BZX810, Nikon Eclipse-Ti, or Olympus IX83).

\section{Dissection of the cap and the ring}

Each cell aggregate containing continuous stratified neuroepithelial tissue was subjected to dissection of the cap and the ring (Process 5). The central part of the neuroepithelial tissue was cut from the cell aggregate under a microscope as described (Shirai et al., 2016). The dissected inner-central neuroepithelial tissue sheet was collected as the cap. At the same time, the surrounding outer-peripheral part of the neuroepithelial tissue was cut from the cell aggregate and harvested as the ring. The obtained caps and rings were subjected to the RT-preservation method and the ring-qPCR test, respectively. Caps whose rings passed the ring-PCR test were used as retinal sheets for transplantation.

\section{qPCR analysis and ring-PCR test}

Rings and other tissue samples were lysed with Buffer RLT (Qiagen) containing 1\% 2mercaptoethanol, and total RNA was extracted and purified using an RNeasy Micro Kit (Qiagen). The total RNA was reverse-transcribed and subjected to qPCR using a 
StepOne Plus Real-Time PCR System (Applied Biosystems) or Biomark HD (Fluidigm) according to the manufacturers' instructions. Based on the Ct values for each gene, Zscores were calculated for the individual caps or rings. Data were visualized as heat maps using the heatmap. 2 function of the R/Bioconductor package gplots. PCA was performed using the prcomp function in the R base package. The PCA results were visualized using R package ggplot2.

\section{Microarray analysis}

Dissected tissues in the cell aggregates were lysed with Buffer RLT (Qiagen) containing 1\% 2-mercaptoethanol, and total RNA was extracted and purified using an RNeasy Micro Kit (Qiagen). Microarray analysis using a GeneChip array was performed by Kurabo Industries (Osaka, Japan). Briefly, total RNA was reverse-transcribed to cDNA with T7 oligo d(T) primer (Affymetrix). Next, biotin-labeled cRNA was synthesized and amplified by in vitro transcription of the second-strand cDNA template using T7 RNA polymerase (Affymetrix). The labeled cRNA was purified and fragmented, loaded onto a GeneChip ${ }^{\circledR}$ Human Genome U133 Plus2.0 array (Affymetrix), and hybridized according to the manufacturer's protocol. Raw intensity data from the GeneChip array were analyzed using GeneChip Operating Software (Affymetrix). The data were logarithmically transformed and $\log \mathrm{FC}$ was calculated for each gene. The $\log \mathrm{FC}$ data were visualized as heat maps using the heatmap. 2 function of the R/Bioconductor package gplots. 


\section{RT-preservation method}

Cell aggregates and tissue sheets (caps) were transferred to $1.5-\mathrm{mL}$ or $15-\mathrm{mL}$ tubes.

After washout of the culture supernatant, preservation solution was added and the tubes were placed in incubators set at $4,12,15,17,20,22$, and $37^{\circ} \mathrm{C}$. For temperature validation, the temperatures of the incubators were monitored by temperature loggers in some experiments. As candidate preservation solutions, Optisol-GS (Bausch \& Lomb), balanced salt solution (BSS) (Gibco), Belzer UW Cold Storage Solution (Bridge to Life), and cell culture media were used. For RT-preservation of caps, each 1.5-mL tube containing a cap in Optisol was placed in a cool incubator (Mitsubishi Electric Engineering) set at $17^{\circ} \mathrm{C}$. Caps that passed QC tests, including the ring-PCR test were picked up and used for transplantation.

\section{Animals, transplantation in rats, and ex vivo electrophysiology assay}

All the animal experimental protocols were approved by the animal care committee of the RIKEN Center for Biosystems Dynamics Research (BDR) and were conducted in accordance with the Association for Research in Vision and Ophthalmology Statement for the Use of Animals in Ophthalmic and Vision Research. SD-Foxn1 Tg(S334ter)3Lav nude rats (RD-nude rats) were obtained from the Rat Resource and Research Center (Sagdullaev et al., 2003; Seiler et al., 2014). Transplantation in the subretinal space of rats was performed as described (Tu et al., 2019). RD-nude rats transplanted with iPSCretinal sheets were used for MEA recordings at 13.5-15 months of age (i.e., 8-10.5 months after transplantation). The MEA recordings were performed using the USB- 
MEA60-Up-System (Multi Channel Systems) as described (Matsuyama et al., 2021; Tu et al., 2019). Briefly, RD-nude rats were dark-adapted for 1-3 days before use. Rats were anesthetized and sacrificed by excess inhalation of Isoflurane or Sevoflurane. Their eyecups were then harvested under dim red light with wavelength peaked at 700 nm and placed in oxygenated Ames' medium (Sigma-Aldrich) in the dark. The retina was carefully isolated from the eyecup and the residual vitreous was removed. The retina was mounted with the RGC side down. The engrafted area was recognized by its dotted appearance and centered on the electrode. Full-field light stimuli with $10 \mathrm{~ms}$ and $1 \mathrm{sec}$ duration at different intensities (weak: $10.56 \log$ photons $/ \mathrm{cm}^{2} / \mathrm{s}$; medium: 12.16 $\log$ photons $/ \mathrm{cm}^{2} / \mathrm{s}$; strong: $12.84 \log$ photons $/ \mathrm{cm}^{2} / \mathrm{s}$ ) were generated using a white LED (NSPW500C; Nichia Corp.). Each set of stimulation, composed of 3 repeats for each stimulation intensity and duration combination, was repeated before addition of L-AP4 (Before), in the presence of $10 \mu \mathrm{M}$ L-AP4 (L-AP4, mGluR6 blocker, Wako), and after washout of L-AP4 (After washout). Super-strong stimuli (15.48 $\log$ photons $/ \mathrm{cm}^{2} / \mathrm{s}$ ) were applied at the end of the MEA recordings to confirm the cell viability in the retinas. The MEA data were collected at a $20-\mathrm{kHz}$ sampling rate without filtering. The recorded spikes were sorted offline to count RGC-spikes using the automatic template formation and spike matching algorithm in Spike 2 (version 7.2; CED) with minor modifications (Matsuyama et al., 2021). RGC light responses were defined as having 2fold increase of spiking frequency against the stimulation onset and/or offset. The average RGC-spike counts and the average RGC light response probability (RGC number with light-responsive spikes per number of recorded RGC) was calculated from 
all detected RGCs in each sample as described (Matsuyama et al., 2021; Tu et al., 2019).

\section{In vivo tumorigenicity study}

In vivo tumorigenicity study using animals was approved by the IRB of the Foundation for Biomedical Research and Innovation (FBRI), the Committee for Animal Experiments of the FBRI, and the animal care committee of RIKEN BDR. Subretinal transplantation was conducted in RIKEN BDR. Six-week-old female nude rats (F344/NJcl-rnu/rnu rats; CLEA Japan) were used for the transplantation and underwent general condition observations and weight measurements during the observation period. Fundus imaging and OCT analysis were performed using RS-3000 Advance (Nidek) and Micron IV and OCT (Phoenix research labs) according to the manufacturers' instructions. After necropsy, the eyeballs were fixed at $4{ }^{\circ} \mathrm{C}$ in SUPERFIX (KY-500; Kurabo Japan), embedded in paraffin, and sliced with a microtome at 3- $\mu$ m thickness. One in every five sections was used for HE staining and histopathological analysis, and the other sections were used for immunohistochemistry.

\section{Immunohistochemistry}

IHC was performed as described (Kuwahara et al., 2019). Cell aggregates and transplanted retinas harvested from RD-nude rats were fixed with 4\% paraformaldehyde (Wako) and sectioned with a cryostat (Leica) to prepare frozen sections. Frozen sections and paraffin sections were treated with or without heat-based antigen retrieval in Target 
Retrieval solution (Dako) at $105^{\circ} \mathrm{C}$ for $15 \mathrm{~min}$. The primary antibodies used were as follows: anti-Chx10 (sheep; 1:500; Exalpha), anti-Pax6 (mouse; 1:1000; BD Biosciences), anti-Rx (guinea pig; 1:2000; Takara), anti-Crx (rabbit; 1:200; Takara), anti-Recoverin (rabbit; 1:500; Proteintech), anti-RXRG (mouse; 1:500; Santa Cruz Biotechnology), anti-NRL (goat; 1:500; R\&D Systems), anti-Lhx2 (rabbit; 1:500; Millipore), anti-S-opsin (rabbit; 1:500; Santa Cruz Biotechnology), anti-L/M-opsin (rabbit; 1:500; Millipore), anti-Cone-arrestin (Arrestin-3; goat; 1:500; Novus), antiRhodopsin (mouse; 1:1000; Sigma; RET-P1), anti-S-arrestin (mouse; 1:500; Novus), anti-CtBP2 (mouse; 1:500; BD Biosciences), anti-PKCalpha (goat; 1:500; R\&D Systems), anti-Ki67 (mouse; 1:500; BD Biosciences), anti-Aqp1 (Aquaporin1; rabbit; 1:500; Millipore), anti-Emx2 (sheep; 1:100; R\&D Systems), anti-cleaved caspase-3 (rabbit; 1:200; Cell Signaling Technology), anti-HuNu (mouse; 1:500; Millipore), antihuman Ku80 (rabbit; 1:500; Cell Signaling Technology), anti-human Ku80 (goat; 1:500; R\&D Systems), anti-Gnat1 (rabbit; 1:500; Santa Cruz Biotechnology), anti-Gnat2 (rabbit; 1:500; Santa Cruz Biotechnology), anti-PRPH2 (mouse; 1:500; Millipore), antiPNA-Alexa Fluor 647 (1:500; Thermo), anti-Synaptophysin (goat; 1:500; R\&D Systems), and anti-LRIT3 (rabbit; 1:500; Novus). Nuclear counterstaining was performed with DAPI (Nacalai). Stained sections were analyzed with a fluorescence microscope (Keyence BZX810) or a laser scanning confocal microscope (Olympus Fluoview FV1000D, Leica TCS SP-8, or Carl Zeiss LSM880). Image analyses were performed with IMARIS (Oxford Instruments), ImageJ (National Institutes of Health), and Zen Blue (Carl Zeiss) imaging software. 


\section{EdU uptake analysis}

For EdU labeling, control retinas before preservation $(\mathrm{d} 70+0)$, retinas preserved in Optisol at $17^{\circ} \mathrm{C}$ for 2 days $(\mathrm{d} 70+2)$ and 4 days $(\mathrm{d} 70+4)$, and retinas recovered in culture at $37{ }^{\circ} \mathrm{C}$ for 3 days $(\mathrm{d} 70+4+3)$ and 7 days $(\mathrm{d} 70+4+7)$ were treated with the thymidine analog EdU for $4 \mathrm{~h}$. The EdU-treated retinas were fixed and sectioned with a cryostat (Leica). Sections of the retinas were stained with Alexa Fluor 488-labeled azide (Invitrogen) according to the manufacturer's protocol. Images were obtained using a confocal laser microscopeand the numbers of EdU-positive cells and DAPI-positive nuclei were counted using ImageJ software (National Institutes of Health).

\section{Statistical analysis}

Statistical analyses were performed with R version 3.6.0 (The R Foundation for Statistical Computing). A two-tailed Student's $t$-test was carried out for two-group comparisons, and one-way analysis of variance (ANOVA) followed by Tukey's test was performed for multiple-group comparisons. Intergroup differences in body weight and survival rate were tested by one-way ANOVA and the log-rank test, respectively.

\section{Data availability}

All reasonable requests for data that are not included in this article or supplementary content will be promptly reviewed by the senior authors to determine whether the request is subject to any intellectual property or confidentiality obligations. Any data 
and materials that can be shared will be released via a contract. This study did not generate new cell lines. 


\section{References}

Assawachananont, J., Mandai, M., Okamoto, S., Yamada, C., Eiraku, M., Yonemura, S., ... Takahashi, M. (2014). Transplantation of embryonic and induced pluripotent stem cell-derived 3D retinal sheets into retinal degenerative mice. Stem Cell Reports, 2(5), 662-674. doi:10.1016/j.stemcr.2014.03.011

Boucherie, C., Mukherjee, S., Henckaerts, E., Thrasher, A. J., Sowden, J. C., \& Ali, R. R. (2013). Brief report: self-organizing neuroepithelium from human pluripotent stem cells facilitates derivation of photoreceptors. STEM CELLS, 31(2), 408-414. doi:10.1002/stem.1268

Capowski, E. E., Samimi, K., Mayerl, S. J., Phillips, M. J., Pinilla, I., Howden, S. E., . . Gamm, D. M. (2019). Reproducibility and staging of 3D human retinal organoids across multiple pluripotent stem cell lines. Development, 146(1). doi:10.1242/dev.171686

Clevers, H. (2016). Modeling Development and Disease with Organoids. Cell, 165(7), 1586-1597. doi:10.1016/j.cell.2016.05.082

Collin, J., Queen, R., Zerti, D., Dorgau, B., Hussain, R., Coxhead, J., . . . Lako, M. (2019). Deconstructing Retinal Organoids: Single Cell RNA-Seq Reveals the Cellular Components of Human Pluripotent Stem Cell-Derived Retina. STEM CELLS, 37(5), 593-598. doi:10.1002/stem.2963

Cowan, C. S., Renner, M., De Gennaro, M., Gross-Scherf, B., Goldblum, D., Hou, Y., .. . Roska, B. (2020). Cell Types of the Human Retina and Its Organoids at Single-Cell Resolution. Cell, 182(6), 1623-1640.e1634. doi:10.1016/j.cell.2020.08.013

Doi, D., Magotani, H., Kikuchi, T., Ikeda, M., Hiramatsu, S., Yoshida, K., . . Takahashi, J. (2020). Pre-clinical study of induced pluripotent stem cell-derived dopaminergic progenitor cells for Parkinson's disease. Nat Commun, 11(1), 3369. doi:10.1038/s41467-020-17165-w

Eiraku, M., Takata, N., Ishibashi, H., Kawada, M., Sakakura, E., Okuda, S., . . Sasai, Y. (2011). Selforganizing optic-cup morphogenesis in three-dimensional culture. Nature, 472(7341), 51-56. doi:10.1038/nature09941

Eiraku, M., Watanabe, K., Matsuo-Takasaki, M., Kawada, M., Yonemura, S., Matsumura, M., . . . Sasai, Y. (2008). Self-organized formation of polarized cortical tissues from ESCs and its active manipulation by extrinsic signals. Cell Stem Cell, 3(5), 519-532. doi:10.1016/j.stem.2008.09.002

Furukawa, T., Morrow, E. M., \& Cepko, C. L. (1997). Crx, a novel otx-like homeobox gene, shows photoreceptor-specific expression and regulates photoreceptor differentiation. Cell, 91(4), 
531-541. doi:10.1016/s0092-8674(00)80439-0

Gagliardi, G., Ben M'Barek, K., Chaffiol, A., Slembrouck-Brec, A., Conart, J. B., Nanteau, C., . . . Goureau, O. (2018). Characterization and Transplantation of CD73-Positive Photoreceptors Isolated from Human iPSC-Derived Retinal Organoids. Stem Cell Reports, 11(3), 665-680. doi:10.1016/j.stemcr.2018.07.005

Gagliardi, G., Ben M'Barek, K., \& Goureau, O. (2019). Photoreceptor cell replacement in macular degeneration and retinitis pigmentosa: A pluripotent stem cell-based approach. Prog Retin Eye Res, 71, 1-25. doi:10.1016/j.preteyeres.2019.03.001

Gavalas, A., Ruhrberg, C., Livet, J., Henderson, C. E., \& Krumlauf, R. (2003). Neuronal defects in the hindbrain of Hoxa1, Hoxb1 and Hoxb2 mutants reflect regulatory interactions among these Hox genes. Development, 130(23), 5663-5679. doi:10.1242/dev.00802

Georgiou, M., Chichagova, V., Hilgen, G., Dorgau, B., Sernagor, E., Armstrong, L., \& Lako, M. (2020). Room temperature shipment does not affect the biological activity of pluripotent stem cellderived retinal organoids. PLoS One, 15(6), e0233860. doi:10.1371/journal.pone.0233860

Gonzalez-Cordero, A., Kruczek, K., Naeem, A., Fernando, M., Kloc, M., Ribeiro, J., . . A Ali, R. R. (2017). Recapitulation of Human Retinal Development from Human Pluripotent Stem Cells Generates Transplantable Populations of Cone Photoreceptors. Stem Cell Reports, 9(3), 820837. doi:10.1016/j.stemcr.2017.07.022

Gonzalez-Cordero, A., West, E. L., Pearson, R. A., Duran, Y., Carvalho, L. S., Chu, C. J., . . Ali, R. R. (2013). Photoreceptor precursors derived from three-dimensional embryonic stem cell cultures integrate and mature within adult degenerate retina. Nat Biotechnol, 31(8), 741-747. doi:10.1038/nbt.2643

Hamann, S., Zeuthen, T., La Cour, M., Nagelhus, E. A., Ottersen, O. P., Agre, P., \& Nielsen, S. (1998). Aquaporins in complex tissues: distribution of aquaporins 1-5 in human and rat eye. Am J Physiol, 274(5), C1332-1345. doi:10.1152/ajpcell.1998.274.5.C1332

Ikeda, H., Osakada, F., Watanabe, K., Mizuseki, K., Haraguchi, T., Miyoshi, H., . . Sasai, Y. (2005). Generation of Rx+/Pax6+ neural retinal precursors from embryonic stem cells. Proc Natl Acad Sci U S A, 102(32), 11331-11336. doi:10.1073/pnas.0500010102

Iraha, S., Tu, H. Y., Yamasaki, S., Kagawa, T., Goto, M., Takahashi, R., . . Mandai, M. (2018). Establishment of Immunodeficient Retinal Degeneration Model Mice and Functional Maturation of Human ESC-Derived Retinal Sheets after Transplantation. Stem Cell Reports, 10(3), 1059-1074. doi:10.1016/j.stemcr.2018.01.032

Ishikawa, J., Oshima, M., Iwasaki, F., Suzuki, R., Park, J., Nakao, K., . . Tsuji, T. (2015). Hypothermic 
temperature effects on organ survival and restoration. Sci Rep, 5, 9563. doi:10.1038/srep09563

Kanemura, H., Go, M. J., Shikamura, M., Nishishita, N., Sakai, N., Kamao, H., . . Kawamata, S. (2014). Tumorigenicity studies of induced pluripotent stem cell (iPSC)-derived retinal pigment epithelium (RPE) for the treatment of age-related macular degeneration. PLoS One, 9(1), e85336. doi:10.1371/journal.pone.0085336

Kawasaki, H., Suemori, H., Mizuseki, K., Watanabe, K., Urano, F., Ichinose, H., . . Sasai, Y. (2002). Generation of dopaminergic neurons and pigmented epithelia from primate ES cells by stromal cell-derived inducing activity. Proc Natl Acad Sci U S A, 99(3), 1580-1585. doi:10.1073/pnas.032662199

Kitahata, S., Tanaka, Y., Hori, K., Kime, C., Sugita, S., Ueda, H., \& Takahashi, M. (2019). Critical Functionality Effects from Storage Temperature on Human Induced Pluripotent Stem CellDerived Retinal Pigment Epithelium Cell Suspensions. Sci Rep, 9(1), 2891. doi:10.1038/s41598-018-38065-6

Kuroda, T., Yasuda, S., Kusakawa, S., Hirata, N., Kanda, Y., Suzuki, K., . . Sato, Y. (2012). Highly sensitive in vitro methods for detection of residual undifferentiated cells in retinal pigment epithelial cells derived from human iPS cells. PLoS One, 7(5), e37342. doi:10.1371/journal.pone.0037342

Kuwahara, A., Ozone, C., Nakano, T., Saito, K., Eiraku, M., \& Sasai, Y. (2015). Generation of a ciliary margin-like stem cell niche from self-organizing human retinal tissue. Nat Commun, 6, 6286. doi:10.1038/ncomms 7286

Kuwahara, A., Yamasaki, S., Mandai, M., Watari, K., Matsushita, K., Fujiwara, M., . . Kimura, T. (2019). Preconditioning the Initial State of Feeder-free Human Pluripotent Stem Cells Promotes Self-formation of Three-dimensional Retinal Tissue. Sci Rep, 9(1), 18936. doi:10.1038/s41598-019-55130-w

Lamba, D. A., Gust, J., \& Reh, T. A. (2009). Transplantation of Human Embryonic Stem Cell-Derived Photoreceptors Restores Some Visual Function in Crx-Deficient Mice. Cell Stem Cell, 4. doi:10.1016/j.stem.2008.10.015

Lamba, D. A., Karl, M. O., Ware, C. B., \& Reh, T. A. (2006). Efficient generation of retinal progenitor cells from human embryonic stem cells. Proc Natl Acad Sci USA, 103. doi:10.1073/pnas.0601990103

Lin, B., McLelland, B. T., Aramant, R. B., Thomas, B. B., Nistor, G., Keirstead, H. S., \& Seiler, M. J. (2020). Retina Organoid Transplants Develop Photoreceptors and Improve Visual Function 
in RCS Rats With RPE Dysfunction. Invest Ophthalmol Vis Sci, 61(11), 34. doi:10.1167/iovs.61.11.34

Liu, I. S., Chen, J. D., Ploder, L., Vidgen, D., van der Kooy, D., Kalnins, V. I., \& McInnes, R. R. (1994). Developmental expression of a novel murine homeobox gene (Chx10): evidence for roles in determination of the neuroretina and inner nuclear layer. Neuron, 13(2), 377-393. doi:10.1016/0896-6273(94)90354-9

Llonch, S., Carido, M., \& Ader, M. (2018). Organoid technology for retinal repair. Dev Biol, 433. doi:10.1016/j.ydbio.2017.09.028

MacLaren, R. E., Pearson, R. A., MacNeil, A., Douglas, R. H., Salt, T. E., Akimoto, M., . . Ali, R. R. (2006). Retinal repair by transplantation of photoreceptor precursors. Nature, 444(7116), 203207. doi:10.1038/nature 05161

Mandai, M., Fujii, M., Hashiguchi, T., Sunagawa, G. A., Ito, S. I., Sun, J., . . Takahashi, M. (2017). iPSC-Derived Retina Transplants Improve Vision in rd1 End-Stage Retinal-Degeneration Mice. Stem Cell Reports, 8(1), 69-83. doi:10.1016/j.stemcr.2016.12.008

Matsuyama, T., Tu, H. Y., Sun, J., Hashiguchi, T., Akiba, R., Sho, J., . . . Mandai, M. (2021). Genetically engineered stem cell-derived retinal grafts for improved retinal reconstruction after transplantation. iScience, 24(8), 102866. doi:10.1016/j.isci.2021.102866

McLelland, B. T., Lin, B., Mathur, A., Aramant, R. B., Thomas, B. B., Nistor, G., . . Seiler, M. J. (2018). Transplanted hESC-Derived Retina Organoid Sheets Differentiate, Integrate, and Improve Visual Function in Retinal Degenerate Rats. Invest Ophthalmol Vis Sci, 59(6), 25862603. doi:10.1167/iovs.17-23646

Means, T. L., Geroski, D. H., L'Hernault, N., Grossniklaus, H. E., Kim, T., \& Edelhauser, H. F. (1996). The corneal epithelium after optisol-GS storage. Cornea, 15(6), 599-605.

Mellough, C. B., Collin, J., Khazim, M., White, K., Sernagor, E., Steel, D. H., \& Lako, M. (2015). IGF-1 Signaling Plays an Important Role in the Formation of Three-Dimensional Laminated Neural Retina and Other Ocular Structures From Human Embryonic Stem Cells. STEM CELLS, 33(8), 2416-2430. doi:10.1002/stem.2023

Mellough, C. B., Sernagor, E., Moreno-Gimeno, I., Steel, D. H. W., \& Lako, M. (2012). Efficient stage-specific differentiation of human pluripotent stem cells toward retinal photoreceptor cells. STEM CELLS, 30. doi:10.1002/stem. 1037

Meyer, J. S., Howden, S. E., Wallace, K. A., Verhoeven, A. D., Wright, L. S., Capowski, E. E., . . . Gamm, D. M. (2011). Optic vesicle-like structures derived from human pluripotent stem cells facilitate a customized approach to retinal disease treatment. STEM CELLS, 29(8), 1206-1218. 
doi:10.1002/stem.674

Meyer, J. S., Shearer, R. L., Capowski, E. E., Wright, L. S., Wallace, K. A., McMillan, E. L., . . . Gamm, D. M. (2009). Modeling early retinal development with human embryonic and induced pluripotent stem cells. Proc Natl Acad Sci U S A, 106(39), 16698-16703. doi:10.1073/pnas.0905245106

Miyazaki, T., Futaki, S., Suemori, H., Taniguchi, Y., Yamada, M., Kawasaki, M., . . Kawase, E. (2012). Laminin E8 fragments support efficient adhesion and expansion of dissociated human pluripotent stem cells. Nat Commun, 3, 1236. doi:10.1038/ncomms2231

Nahar, S., Nakashima, Y., Miyagi-Shiohira, C., Kinjo, T., Kobayashi, N., Saitoh, I., . . Fujita, J. (2018). A Comparison of the Preservation of Mouse Adipose Tissue-Derived Mesenchymal Stem Cells Using the University of Wisconsin Solution and Hank's Balanced Salt Solution. Stem Cells Int, 2018, 1625464. doi:10.1155/2018/1625464

Nakagawa, M., Taniguchi, Y., Senda, S., Takizawa, N., Ichisaka, T., Asano, K., . . Yamanaka, S. (2014). A novel efficient feeder-free culture system for the derivation of human induced pluripotent stem cells. Sci Rep, 4, 3594. doi:10.1038/srep03594

Nakano, T., Ando, S., Takata, N., Kawada, M., Muguruma, K., Sekiguchi, K., . . Sasai, Y. (2012). Self-formation of optic cups and storable stratified neural retina from human ESCs. Cell Stem Cell, 10(6), 771-785. doi:10.1016/j.stem.2012.05.009

Osakada, F., Ikeda, H., Mandai, M., Wataya, T., Watanabe, K., Yoshimura, N., . . . Takahashi, M. (2008). Toward the generation of rod and cone photoreceptors from mouse, monkey and human embryonic stem cells. Nat Biotechnol, 26(2), 215-224. doi:10.1038/nbt1384

Pearson, R. A., Barber, A. C., Rizzi, M., Hippert, C., Xue, T., West, E. L., . . Ali, R. R. (2012). Restoration of vision after transplantation of photoreceptors. Nature, 485(7396), 99-103. doi:10.1038/nature 10997

Pearson, R. A., Gonzalez-Cordero, A., West, E. L., Ribeiro, J. R., Aghaizu, N., Goh, D., .. . Ali, R. R. (2016). Donor and host photoreceptors engage in material transfer following transplantation of post-mitotic photoreceptor precursors. Nat Commun, 7, 13029. doi:10.1038/ncomms 13029

Phillips, M. J., Jiang, P., Howden, S., Barney, P., Min, J., York, N. W., . . . Gamm, D. M. (2018). A Novel Approach to Single Cell RNA-Sequence Analysis Facilitates In Silico Gene Reporting of Human Pluripotent Stem Cell-Derived Retinal Cell Types. STEM CELLS, 36(3), 313-324. doi:10.1002/stem.2755

Radtke, N. D., Aramant, R. B., Petry, H. M., Green, P. T., Pidwell, D. J., \& Seiler, M. J. (2008). Vision improvement in retinal degeneration patients by implantation of retina together with retinal 
pigment epithelium. Am J Ophthalmol, 146(2), 172-182. doi:10.1016/j.ajo.2008.04.009

Reichman, S., Slembrouck, A., Gagliardi, G., Chaffiol, A., Terray, A., Nanteau, C., . . Goureau, O. (2017). Generation of Storable Retinal Organoids and Retinal Pigmented Epithelium from Adherent Human iPS Cells in Xeno-Free and Feeder-Free Conditions. STEM CELLS, 35(5), 1176-1188. doi:10.1002/stem.2586

Reichman, S., Terray, A., Slembrouck, A., Nanteau, C., Orieux, G., Habeler, W., . . . Goureau, O. (2014). From confluent human iPS cells to self-forming neural retina and retinal pigmented epithelium. Proc Natl Acad Sci U S A, 111(23), 8518-8523. doi:10.1073/pnas.1324212111

Ribeiro, J., Procyk, C. A., West, E. L., O'Hara-Wright, M., Martins, M. F., Khorasani, M. M., . . Ali, R. R. (2021). Restoration of visual function in advanced disease after transplantation of purified human pluripotent stem cell-derived cone photoreceptors. Cell Rep, 35(3), 109022. doi:10.1016/j.celrep.2021.109022

Sagdullaev, B. T., Aramant, R. B., Seiler, M. J., Woch, G., \& McCall, M. A. (2003). Retinal transplantation-induced recovery of retinotectal visual function in a rodent model of retinitis pigmentosa. Invest Ophthalmol Vis Sci, 44. doi:10.1167/iovs.02-0615

Sakaguchi, H., Kadoshima, T., Soen, M., Narii, N., Ishida, Y., Ohgushi, M., . . Sasai, Y. (2015). Generation of functional hippocampal neurons from self-organizing human embryonic stem cell-derived dorsomedial telencephalic tissue. Nat Commun, 6, 8896. doi:10.1038/ncomms9896

Santos-Ferreira, T., Llonch, S., Borsch, O., Postel, K., Haas, J., \& Ader, M. (2016). Retinal transplantation of photoreceptors results in donor-host cytoplasmic exchange. Nat Commun, 7, 13028. doi:10.1038/ncomms 13028

Sasai, Y. (2013). Next-generation regenerative medicine: organogenesis from stem cells in 3D culture. Cell Stem Cell, 12(5), 520-530. doi:10.1016/j.stem.2013.04.009

Sato, T., Vries, R. G., Snippert, H. J., van de Wetering, M., Barker, N., Stange, D. E., . . Clevers, H. (2009). Single Lgr5 stem cells build crypt-villus structures in vitro without a mesenchymal niche. Nature, 459(7244), 262-265. doi:10.1038/nature07935

Seiler, M. J., \& Aramant, R. B. (2012). Cell replacement and visual restoration by retinal sheet transplants. Prog Retin Eye Res, 31(6), 661-687. doi:10.1016/j.preteyeres.2012.06.003

Seiler, M. J., Aramant, R. B., Jones, M. K., Ferguson, D. L., Bryda, E. C., \& Keirstead, H. S. (2014). A new immunodeficient pigmented retinal degenerate rat strain to study transplantation of human cells without immunosuppression. Graefes Arch Clin Exp Ophthalmol, 252(7), 10791092. doi:10.1007/s00417-014-2638-y 
Shirai, H., Mandai, M., Matsushita, K., Kuwahara, A., Yonemura, S., Nakano, T., . . Takahashi, M. (2016). Transplantation of human embryonic stem cell-derived retinal tissue in two primate models of retinal degeneration. Proc Natl Acad Sci $U S$ A, 113(1), E81-90. doi:10.1073/pnas. 1512590113

Singh, M. S., Balmer, J., Barnard, A. R., Aslam, S. A., Moralli, D., Green, C. M., . . MacLaren, R. E. (2016). Transplanted photoreceptor precursors transfer proteins to host photoreceptors by a mechanism of cytoplasmic fusion. Nat Commun, 7, 13537. doi:10.1038/ncomms13537

Sridhar, A., Hoshino, A., Finkbeiner, C. R., Chitsazan, A., Dai, L., Haugan, A. K., . . Reh, T. A. (2020). Single-Cell Transcriptomic Comparison of Human Fetal Retina, hPSC-Derived Retinal Organoids, and Long-Term Retinal Cultures. Cell Rep, 30(5), 1644-1659.e1644. doi:10.1016/j.celrep.2020.01.007

Stamer, W. D., Bok, D., Hu, J., Jaffe, G. J., \& McKay, B. S. (2003). Aquaporin-1 channels in human retinal pigment epithelium: role in transepithelial water movement. Invest Ophthalmol Vis Sci, 44(6), 2803-2808. doi:10.1167/iovs.03-0001

Suga, H., Kadoshima, T., Minaguchi, M., Ohgushi, M., Soen, M., Nakano, T., . . Sasai, Y. (2011). Self-formation of functional adenohypophysis in three-dimensional culture. Nature, 480(7375), 57-62. doi:10.1038/nature 10637

Sugai, K., Sumida, M., Shofuda, T., Yamaguchi, R., Tamura, T., Kohzuki, T., . . Okano, H. (2021). First-in-human clinical trial of transplantation of iPSC-derived NS/PCs in subacute complete spinal cord injury: Study protocol. Regen Ther, 18, 321-333. doi:10.1016/j.reth.2021.08.005

Takebe, T., \& Wells, J. M. (2019). Organoids by design. Science, 364(6444), 956-959. doi:10.1126/science.aaw7567

Theil, T., Aydin, S., Koch, S., Grotewold, L., \& Rüther, U. (2002). Wnt and Bmp signalling cooperatively regulate graded Emx2 expression in the dorsal telencephalon. Development, 129(13), 3045-3054.

Tu, H. Y., Watanabe, T., Shirai, H., Yamasaki, S., Kinoshita, M., Matsushita, K., . . Mandai, M. (2019). Medium- to long-term survival and functional examination of human iPSC-derived retinas in rat and primate models of retinal degeneration. EBioMedicine, 39, 562-574. doi:10.1016/j.ebiom.2018.11.028

Wahlin, K. J., Cheng, J., Jurlina, S. L., Jones, M. K., Dash, N. R., Ogata, A., . . Zack, D. J. (2021). CRISPR Generated SIX6 and POU4F2 Reporters Allow Identification of Brain and Optic Transcriptional Differences in Human PSC-Derived Organoids. Front Cell Dev Biol, 9, 764725. doi:10.3389/fcell.2021.764725 
Wahlin, K. J., Maruotti, J. A., Sripathi, S. R., Ball, J., Angueyra, J. M., Kim, C., . . Zack, D. J. (2017). Photoreceptor Outer Segment-like Structures in Long-Term 3D Retinas from Human Pluripotent Stem Cells. Sci Rep, 7(1), 766. doi:10.1038/s41598-017-00774-9

Yamasaki, S., Sugita, S., Horiuchi, M., Masuda, T., Fujii, S., Makabe, K., . . Mandai, M. (2021). Low Immunogenicity and Immunosuppressive Properties of Human ESC- and iPSC-Derived Retinas. Stem Cell Reports, 16(4), 851-867. doi:10.1016/j.stemcr.2021.02.021

Zhai, J., Lin, H., Canete-Soler, R., \& Schlaepfer, W. W. (2005). HoxB2 binds mutant SOD1 and is altered in transgenic model of ALS. Hum Mol Genet, 14(18), 2629-2640. doi:10.1093/hmg/ddi297

Zhong, X., Gutierrez, C., Xue, T., Hampton, C., Vergara, M. N., Cao, L. H., . . Canto-Soler, M. V. (2014). Generation of three-dimensional retinal tissue with functional photoreceptors from human iPSCs. Nat Commun, 5, 4047. doi:10.1038/ncomms5047

Zhu, J., Cifuentes, H., Reynolds, J., \& Lamba, D. A. (2017). Immunosuppression via Loss of IL2rgamma Enhances Long-Term Functional Integration of hESC-Derived Photoreceptors in the Mouse Retina. Cell Stem Cell, 20. doi:10.1016/j.stem.2016.11.019 


\section{Acknowledgements}

We are grateful to the CiRA (Kyoto, Japan) and CiRA Foundation (Kyoto, Japan) for kindly providing iPSCs. We thank Dr. Mototsugu Eiraku for advice on retinal maturation culture, and Yasushi Hiramine, Miki Iwata, Kazunari Tanaka, Masahiro Yahata, Koichiro Manabe, Kiyoko Bando, Jiro Akimaru, Keigo Kawabe, Atsushi Tsuchida, Tomokazu Nagano, and members of RACMO for fruitful discussions. We thank Ayumi Kiso for in vivo studies, Kohei Kanata for IHC staining, and Yukiko Ishigami for dissection. A.Ku. would like to express deep gratitude to his late mentor Dr. Yoshiki Sasai, a gifted scientist who pioneered self-organizing stem cell biology. This work was supported by the Research Center Network for Realization of Regenerative Medicine from the Japan Agency for Medical Research and Development (AMED) (M.T., S.K.).

\section{Competing interests}

This work was funded by AMED under grant number JP21bm0204002 (M.T., S.K.) and by Sumitomo Dainippon Pharma Co., Ltd. (Sumitomo). K.W., S.Y., T.Ka., H.A., T.T., Y.K., A.N., K.U., K.O., M.F., Y.H., A.T., R.H., O.T., O.O., H.O., T.H., A.I., D.N., K.M., A.Ki., and A.Ku. are employed by Sumitomo. T.Ki. is a board member of Sumitomo. S.K. has a scientific consulting role for Sumitomo. M.T. and M.M. have received research funding from Sumitomo. The authors are co-inventors on patent applications.

\section{Author contributions}


K.W.: Formal analysis; Investigation; Methodology; Writing - original draft; Designed ring-PCR test and RT-preservation studies. S.Y.: Formal analysis; Investigation; Methodology; Writing - original draft; Designed self-organizing culture and in vivo IHC studies. H.Y.T.: Formal analysis; Investigation; Writing - original draft; Designed electrophysiology assay. M.S.: Formal analysis; Investigation; Writing -original draft; Designed in vivo tumorigenicity study. T.Ka.: Formal analysis; Investigation; Writing original draft; Designed in vivo tumorigenicity study. H.A.: Formal analysis; Investigation; Writing - original draft; Designed in vivo tumorigenicity study. T.T.: Formal analysis; Investigation; Writing - original draft; Designed in vivo tumorigenicity study. Y.K.: Formal analysis; Investigation; Writing -original draft; Designed in vivo tumorigenicity study. A.N.: Investigation. K.U.: Investigation. K.O.: Investigation. C.M.: Investigation. T.M.: Formal analysis; Investigation. J.S.: Investigation. M.N.: Investigation. M.F.: Investigation. Y.H.: Investigation. A.T.: Investigation. R.H.: Investigation. O.T.: Investigation. O.O.: Investigation. H.O.: Investigation. T.H.: Investigation. A.I.: Supervision. D.N.: Investigation. K.M.: Investigation. M.T.: Supervision. A.Ki: Supervision. T.Ki.: Supervision. S.K.: Formal analysis; Supervision; Writing - original draft; Project administration; Designed in vivo tumorigenicity study. M.M.: Conceptualization; Formal analysis; Supervision; Investigation; Writing original draft; Project administration; Designed in vivo efficacy study. A. Ku.: Conceptualization; Formal analysis; Supervision; Investigation; Methodology; Writing original draft; Project administration; Designed self-organizing culture, ring-PCR test, and RT-preservation studies. 


\section{Figure legends}

\section{Figure 1. Self-organizing culture of human iPSCs to generate the 3D-retina and dissected retinal sheet.}

(A) Scheme of the self-organizing culture. Process 1: maintenance culture to expand undifferentiated human iPSCs. Process 2: retinal differentiation from iPSCs to immature retinal tissue. Process 3: induction-reversal culture to generate an induction-reversal intermediate containing retinal tissue. Process 4: maturation culture to generate a 3Dretina with multilayered continuous retinal tissue. Process 5: dissection of the 3D-retina to generate the retinal sheet (final product).

(B) Bright-field view of iPSC-S17-derived cell aggregate containing retinal tissue on days 14, 42, 91, and 180 (upper). Scale bar in bright-field view: $100 \mu \mathrm{m}$.

Immunostaining of iPSC-S17-derived retinal tissue on days 14, 42, 91, and 180 (middle and lower). Crx (green) and Chx 10 (red) in middle panels. Pax6 (green) and Recoverin (red) in lower panels. Blue: nuclear staining with DAPI. Scale bar in immunostaining: $20 \mu \mathrm{m}$.

(C) Immunostaining of iPSC-S17-derived retinal tissue on day 180. RXRG (green) and NRL (red) in upper-left panel. Lhx2 (red) in upper-right panel. CtBP2 (green) and Recoverin (red) in lower-left panel. Cone-arrestin (green) in lower-right panel. Blue: nuclear staining with DAPI. Scale bar: $20 \mu \mathrm{m}$.

(D) Gene expression analysis in iPSC-S17-derived cell aggregates containing retinal tissue on days $0,6,14,45,60$, and 80 . mRNA levels were determined by qPCR analysis. Relative mRNA expression was determined by the delta-delta Ct method with GAPDH as an endogenous control. Data are presented as mean $\pm \operatorname{SE}(n=48$ aggregates per time point).

(E) Bright-field view of iPSC-S17-derived retinal sheet. Scale bar: $100 \mu \mathrm{m}$.

(F) Immunostaining of iPSC-S17-derived retinal sheet on day 87. Crx (green) and Chx10 (red) in left panel. Rx (green) and Recoverin (red) in right panel. Blue: nuclear staining with DAPI. Scale bar: $100 \mu \mathrm{m}$.

\section{Figure 2. Characterization of major off-target tissues in the retinal differentiation culture.}

(A) Bright-field view of retinal tissue and off-target tissues in iPSC-LPF11-derived cell 
aggregates. Scale bar: $100 \mu \mathrm{m}$.

(B) Proportions of cell aggregates containing retinal tissue and off-target tissues. Three independent batches derived from iPSC-LPF11 cells and three independent batches derived from iPSC-S17 cells were analyzed ( $n=59-95$ aggregates per each batch). Data are presented as mean $\pm \mathrm{SE}$.

(C) Heat map for gene expressions in retinal tissue and off-target tissues derived from iPSC-LPF11 cells. As a control, gene expressions in undifferentiated iPSC-LPF11 cells are also shown. Gene expressions were measured by qPCR analyses. Row Z-scores were calculated for each tissue and plotted as a heat map.

(D) Representative bright-field images and IHC images for off-target tissue-1 and -2 derived from iPSC-LPF11 cells. The bright-field images and IHC images were captured at the same positions. Immunostaining for Aqp1 (upper right; red) and Emx2 (lower right; red) is shown. Blue: nuclear staining with DAPI. Scale bar: $100 \mu \mathrm{m}$.

(E) Heat map for gene expressions in retinal tissue and off-target tissue-3 derived from iPSC-1231A3 cells. mRNA levels were determined by microarray analysis. LogFC values were calculated for each gene and plotted as a heat map. FC, fold change. Note that off-target tissue-3 expressed Hox genes compared with retinal tissue.

\section{Figure 3. QC method for retinal sheets by testing gene expression in the surrounding outer tissue-sheet.}

(A) Scheme for dissection of the cap and the ring and the ring-PCR test in Process 5 (dissection).

(B) Bright-field (left) and IHC (middle, right) images of a representative cap and ring. The cap and ring were derived from iPSC-LPF11 cells. Immunostaining for Crx (middle, green), Chx10 (middle, red), Pax6 (right, green), and Recoverin (right, red) is shown. DAPI is shown in blue. Scale bar: $100 \mu \mathrm{m}$.

(C) Heat map for gene expressions in caps and rings derived from iPSC-LPF11 cells. As a control, gene expressions in undifferentiated iPSC-LPF11 cells are also shown. Gene expressions were measured by qPCR analyses. Row Z-scores were calculated for each cap and ring, and plotted as a heat map.

(D) Representative gene expressions in cap and ring dissected from the same aggregate shown as a plot with a regression line (left). The coefficient of determination $\left(\mathrm{R}^{2}\right)$ for the gene expression between each cap and ring is shown (right). \#1-\#6 correspond to 
Tissue \#1-\#6 in (C).

\section{Figure 4. Controlled room temperature non-freezing preservation method.}

(A) Screening for the non-freezing preservation conditions. +++: multilayered continuous retinal epithelium was preserved. ++: multilayered continuous retinal epithelium was preserved but neural rosettes appeared. +: multilayered continuous retinal epithelium was observed but very limited. -, no multilayered continuous retinal epithelium was observed after the preservation.

(B) Scheme of the preservation experiments. iPSC-retinas were preserved in Optisol at $17^{\circ} \mathrm{C}$ for 3-4 days followed by recovery culture at $37^{\circ} \mathrm{C}$ under $\mathrm{CO}_{2}$ for 7 days.

Recovered retinas were subjected to experiments.

(C) Proportions of $\mathrm{Crx}^{+}$photoreceptor precursors in multilayered retinal tissues. The proportions were calculated by dividing the number of $\mathrm{Crx}^{+}$cells by the number of nuclei (DAPI). Data are presented as mean \pm SE for $n=5\left(4{ }^{\circ} \mathrm{C}\right), 4\left(12{ }^{\circ} \mathrm{C}\right), 4\left(15^{\circ} \mathrm{C}\right)$, $5\left(17^{\circ} \mathrm{C}\right), 5\left(20^{\circ} \mathrm{C}\right), 5\left(22^{\circ} \mathrm{C}\right), 4\left(37^{\circ} \mathrm{C}\right)$, and 3 (control culture).

(D) Cell viability in non-preserved retinas (Control) and retinas preserved for 4 days.

Data are shown as mean $\pm \mathrm{SE}$ ( $n=5$ per group).

(E) Gene expressions of Chx 10 and Crx in non-preserved retinas (Control) and retinas preserved for 4 days, determined by qPCR analyses. Data are presented as mean $\pm \mathrm{SE}$ ( $n=3$ per group).

(F) IHC analysis of retinas that were not preserved (d70+0), preserved in Optisol for 2 days $(\mathrm{d} 70+2)$ and 4 days $(\mathrm{d} 70+4)$, and preserved in Optisol for 4 days followed by recovery culture for 3 days $(\mathrm{d} 70+4+3)$ and 7 days $(\mathrm{d} 70+4+7)$. Ki67 (red) staining in the upper panels. EdU staining (green) in lower panels. DAPI is shown in blue. Scale bar: $50 \mu \mathrm{m}$.

(G) Numbers of EdU-positive cells per $100 \mu \mathrm{m}$-wide multilayered retinal tissue in each group. Data are presented as mean $\pm \mathrm{SE}$ ( $n=6$ per group). ${ }^{* * *} p<0.001$ for one-way ANOVA followed by a Tukey's test.

(H) Scheme for dissection, ring-PCR test, and shipping of retinal sheets. Dissected caps are preserved at $17^{\circ} \mathrm{C}$. During the preservation, the corresponding rings are subjected to the ring-qPCR test. Caps whose rings pass the ring-PCR test can be shipped as retinal sheets for transplantation.

n.s., not significant. 
Figure 5. In vivo tumorigenicity study by subretinal transplantation in nude rats. (A, B) Time courses of body weight (A) and Kaplan-Meier survival curves (B) in nude rats in the tumorigenicity study. Intact: control nude rat without surgery $(n=4)$. Sham: control nude rat with subretinal injection of vehicle control $(n=4)$. Transplanted: nude rat with subretinal transplantation of a single iPSC-Q-derived retinal sheet $(n=4)$. Data are presented as mean $\pm \mathrm{SE}$.

(C, D) HE staining of transplanted eye sections at 13, 26, 52, and 78 weeks after transplantation. Scale bar: $100 \mu \mathrm{m}$.

(E-I) Immunostaining of transplanted eye sections for human nuclear markers, retinal markers, and Ki67. Scale bar: $20 \mu \mathrm{m}$. (E) Immunostaining for $\mathrm{HuNu}$ (green), Recoverin (red), Chx10 (white), and DAPI (blue). (F) Immunostaining for human Ku80 (green), Ki67 (red), and DAPI (blue). (G) High-magnification image of immunostaining for a graft in the vitreous cavity (52 weeks). HuNu (green), Recoverin (red), Chx 10 (white), and DAPI (blue). (H) High-magnification image of immunostaining for the graft (52 weeks) with antibodies for human Ku80 (green), Ki67 (red), and DAPI (blue). Arrow indicates $\mathrm{Ki} 67^{+}$and $\mathrm{Ku} 80^{+}$cells. (I) Percentages of $\mathrm{Ki} 67^{+}$and $\mathrm{Ku} 80^{+}$cells among the $\mathrm{Ku} 80^{+}$human cells. Data are presented as mean.

INL, inner nuclear layer.

Figure 6. Engraftment and photoreceptor maturation of iPSC-retinal sheets after subretinal transplantation in RD-nude rats.

(A-J') Immunostaining of rat eyes transplanted with iPSC-S17-derived retinal sheets. The retinal sheets were transplanted in the subretinal space of $\mathrm{RD}$-nude rats. The rat retinas were fixed at 263 days after transplantation (341 days after initiation of differentiation).

(A, A') Immunostaining for human Ku80 (green), Recoverin (red), and PKCalpha (white). Control non-transplanted area (A). Engrafted area (A').

(B-B") Immunostaining for human Ku80 (green), S-arrestin (red), and Cone-arrestin (white). High magnification in (B') and higher magnification in (B'). Boxed area in (B) corresponds to $\left(\mathrm{B}^{\prime}\right)$.

(C-I') Immunostaining for photoreceptor markers. NRL (green), Recoverin (red), and RXRG (white) in (C, C'). S-opsin (green) and L/M-opsin (red) in (D). GNAT1 (green) 
and PRPH2 (red) in (E, E'). GNAT2 (green) and PNA (red) in (F). Human Ku80 (green), Synaptophysin (red), and PKCalpha (white) in (G-G”). Arrowheads in (G', G”): Synaptophysin-positive neurites in no nuclear space. Human Ku80 (green), Calbindin (red), and S-arrestin (white) in (H, $\left.\mathrm{H}^{\prime}\right)$. Arrowheads in $\left(\mathrm{H}^{\prime}\right)$ : Calbindinpositive neurites.

(I, I') Maximum projection image of Z-stacks immunostained for human Ku80 (green),

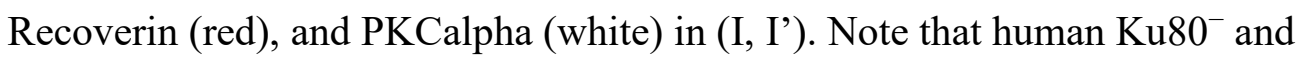
$\mathrm{PKCalpha}^{+}$bipolar cells were located near the human $\mathrm{Ku} 80^{+}$and Recoverin ${ }^{+}$ photoreceptors (arrowheads).

(J, J') Maximum projection image of Z-stacks stained for CtBP2 (green), LRIT3 (red), and PKCalpha (white). High magnification in $\left(\mathrm{J}^{\prime}\right)$. Note that photoreceptor-synapse marker CtBP2 and LRIT3 were expressed near the neurites of $\mathrm{PKCalpha}^{+}$rod bipolar cells.

DAPI staining (blue) in (A, A', B-B', C, D, E, E', F, G, G', H, I, J, J'). Scale bars: 100 $\mu \mathrm{m}$ in (A', B), $10 \mu \mathrm{m}$ in (A, B', B', C-J), and $1 \mu \mathrm{m}$ in (J').

INL, inner nuclear layer; GCL, ganglion cell layer.

\section{Figure 7. Light responses in transplanted iPSC-retinas by ex vivo electrophysiology assay.}

(A) Scheme of ex vivo electrophysiology assay using MEA to evaluate the light responses in transplanted iPSC-retinas. iPSC-S17-derived retinal sheets were transplanted in the subretinal space in RD-nude rats. Transplanted and non-transplanted rat retinas were mounted on the MEA electrodes for electrophysiological recording. During the recording, light stimuli were conducted in different intensities (weak, medium, strong), and the rat retinas were incubated in Ames' medium (Before), followed by addition of L-AP4 (L-AP4), and washout of L-AP4 (After washout). (B-D) Representative results of MEA analysis. (B) Differential interference contrast (DIC) image of a RD-nude rat retina mounted on the MEA. (C, D) Peri-stimulus time histograms to examine the RGC-spike counts during strong light stimuli (12.84 log photons $/ \mathrm{cm}^{2} / \mathrm{s}$ ). The $\mathrm{X}$-axis and $\mathrm{Y}$-axis represent the time and average RGC-spike count, respectively. Recording data for iPSC-retinal sheet-transplanted rat retinas (Transplanted; upper) and non-transplanted rat retinas (Non-transplanted; lower) in the three conditions (Before, L-AP4, After washout) are shown. Multiple electrode 
recording data are shown in (C) and representative data (red box in C) are shown in (D). Yellow box in (C, D): light stimuli. Red arrows in (D): start timing of light stimuli.

(E) RGC light response probabilities. Control non-transplanted rat retinas (Control; $n=$ 12) and iPSC-S17-derived retinal sheet-transplanted rat retinas (Transplanted; $n=12$ ) were subjected to MEA analysis to evaluate the RGC responses to light stimulation. Dots and bars show the per sample (recorded rat retina) summary of the collected data. Dot: RGC light response probability estimated by statistical modeling (Bayesian statistical inference with Markov Chain Monte Carlo sampling). Bar: 89\% confidence interval. 
Figure 1

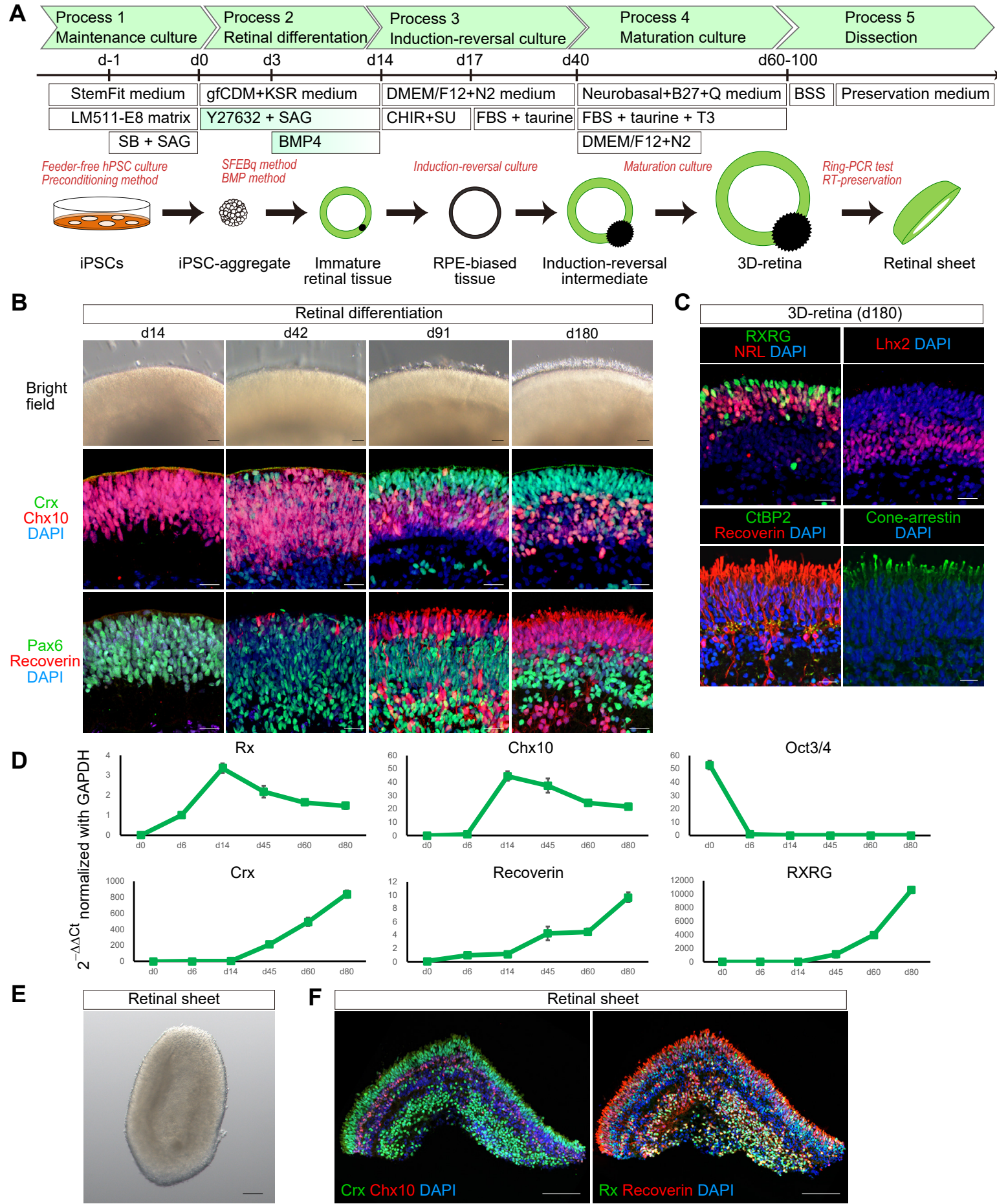

Figure 1. Self-organizing culture of human iPSCs to generate the 3D-retina and dissected retinal sheet.

(A) Scheme of the self-organizing culture. Process 1: maintenance culture to expand undifferentiated human iPSCs. Process 2: retinal differentiation from iPSCs to immature retinal tissue. Process 3: induction-reversal culture to generate an induction-reversal intermediate containing retinal tissue. Process 4: maturation culture to generate a 3D-retina with multilayered continuous retinal tissue. Process 5: dissection of the 3D-retina to generate the retinal sheet (final product).

(B) Bright-field view of iPSC-S17-derived cell aggregate containing retinal tissue on days 14, 42, 91, and 180 (upper). Scale bar in bright-field view: $100 \mu \mathrm{m}$. Immunostaining of iPSC-S17-derived retinal tissue on days 14, 42, 91, and 180 (middle and lower). Crx (green) and Chx10 (red) in middle panels. Pax6 (green) and Recoverin (red) in lower panels. Blue: nuclear staining with DAPI. Scale bar in immunostaining: $20 \mu \mathrm{m}$.

(C) Immunostaining of iPSC-S17-derived retinal tissue on day 180. RXRG (green) and NRL (red) in upper-left panel. Lhx2 (red) in upper-right panel. CtBP2 (green) and Recoverin (red) in lower-left panel. Cone-arrestin (green) in lower-right panel. Blue: nuclear staining with DAPI. Scale bar: 20 $\mu \mathrm{m}$. (D) Gene expression analysis in iPSC-S17-derived cell aggregates containing retinal tissue on days $0,6,14,45,60$, and 80. mRNA levels were determined by qPCR analysis. Relative mRNA expression was determined by the $\triangle \Delta \mathrm{Ct}$ method with GAPDH as an endogenous control. Data are presented as mean \pm SE ( $n=48$ aggregates per time point). (E) Bright-field view of iPSC-S17-derived retinal sheet. Scale bar: $100 \mu \mathrm{m}$.

(F) Immunostaining of iPSC-S17-derived retinal sheet on day 87. Crx (green) and Chx10 (red) in left panel. Rx (green) and Recoverin (red) in right panel. Blue: nuclear staining with DAPI. Scale bar: $100 \mu \mathrm{m}$. 


\section{Figure 2}

A
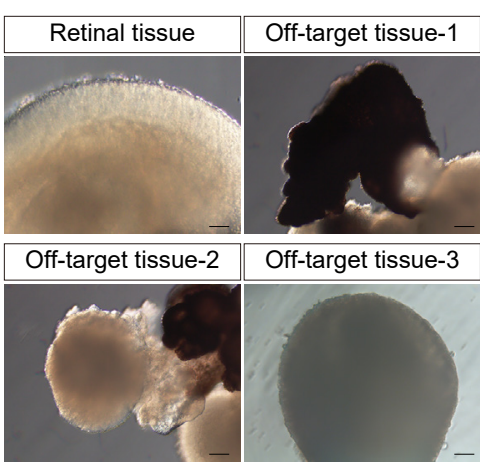

B

Proportions of cell aggregates containing the retinal tissue and off-target tissues

\begin{tabular}{lcccc}
\hline \multirow{2}{*}{ Batch } & \multirow{2}{*}{ \% Retinal tissue } & \multicolumn{3}{c}{$\%$ Off-target tissue } \\
\cline { 3 - 5 } & & 1 & 2 & 3 \\
\hline \#1 LPF11 & 99 & 20 & 7 & 1 \\
\#2 LPF11 & 97 & 39 & 7 & 8 \\
\#3 LPF11 & 100 & 7 & 2 & 0 \\
\#4 S17 & 98 & 17 & 3 & 2 \\
\#5 S17 & 100 & 20 & 0 & 2 \\
\#6 S17 & 100 & 20 & 3 & 2 \\
\hline Average \pm SE & $99 \pm 1$ & $20 \pm 4$ & $4 \pm 1$ & $2 \pm 1$ \\
\hline
\end{tabular}

C

Gene expression analysis of retinal and off-target tissues

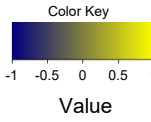

(Row Z-score)

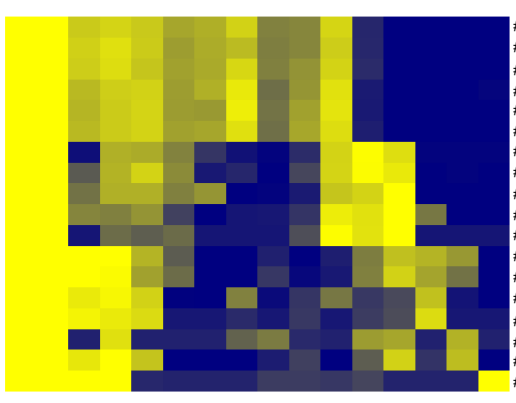

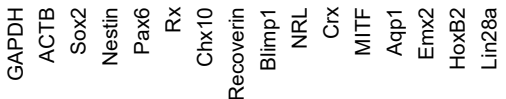
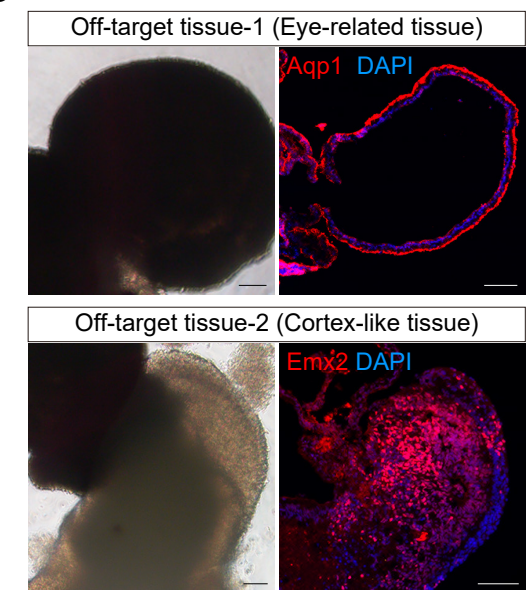

E

Gene expression analysis of retinal tissue and off-target tissue-3
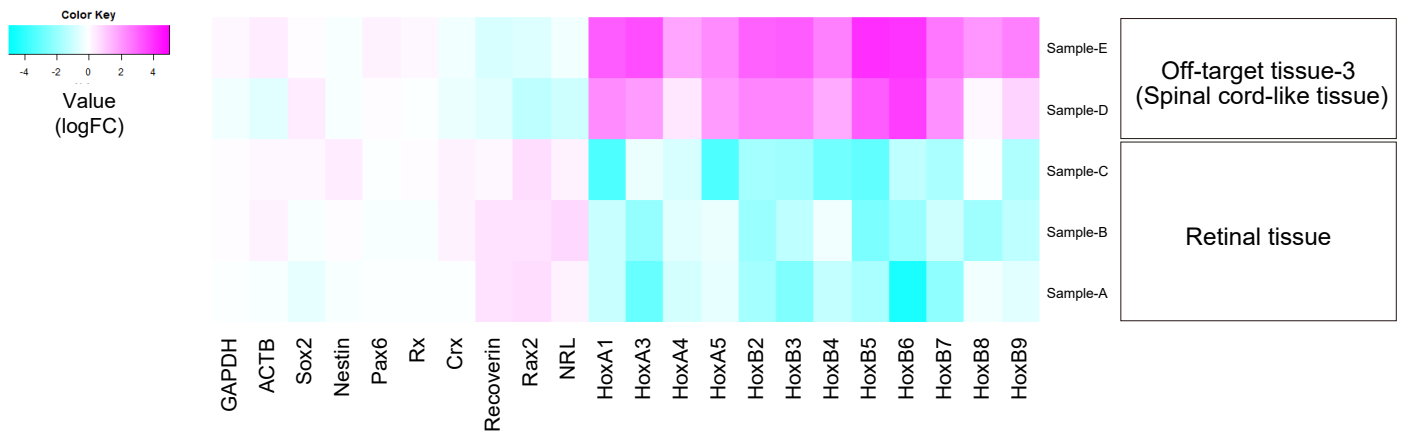

Figure 2. Characterization of major off-target tissues in the retinal differentiation culture.

(A) Bright-field view of retinal tissue and off-target tissues in iPSC-LPF11-derived cell aggregates. Scale bar: $100 \mu \mathrm{m}$.

(B) Proportions of cell aggregates containing retinal tissue and off-target tissues. Three independent batches derived from iPSC-LPF11 cells and three independent batches derived from iPSC-S17 cells were analyzed ( $n=59-95$ aggregates per each batch). Data are presented as mean \pm SE.

(C) Heat map for gene expressions in retinal tissue and off-target tissues derived from iPSC-LPF11 cells. As a control, gene expressions in undifferentiated iPSC-LPF11 cells are also shown. Gene expressions were measured by qPCR analyses. Row Z-scores were calculated for each tissue and plotted as a heat map.

(D) Representative bright-field images and IHC images for off-target tissue-1 and -2 derived from iPSC-LPF11 cells. The bright-field images and IHC images were captured at the same positions. Immunostaining for Aqp1 (upper right; red) and Emx2 (lower right; red) is shown. Blue: nuclear staining with DAPI. Scale bar: $100 \mu \mathrm{m}$.

(E) Heat map for gene expressions in retinal tissue and off-target tissue-3 derived from iPSC-1231A3 cells. mRNA levels were determined by microarray analysis. LogFC values were calculated for each gene and plotted as a heat map. FC, fold change. Note that off-target tissue-3 expressed Hox genes compared with retinal tissue. 
Figure 3

A Retinal tissue with neuroepithelium structure

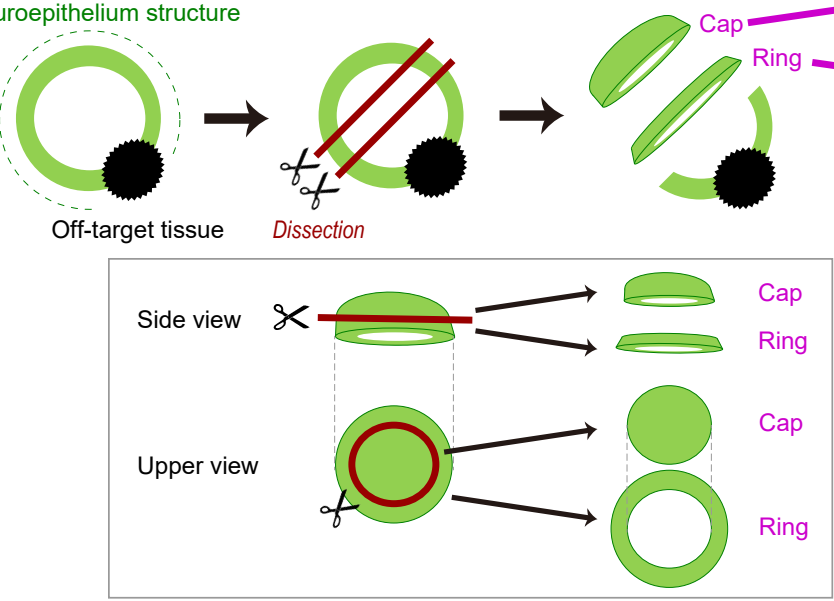

Dissected retinal tissue-sheet

B

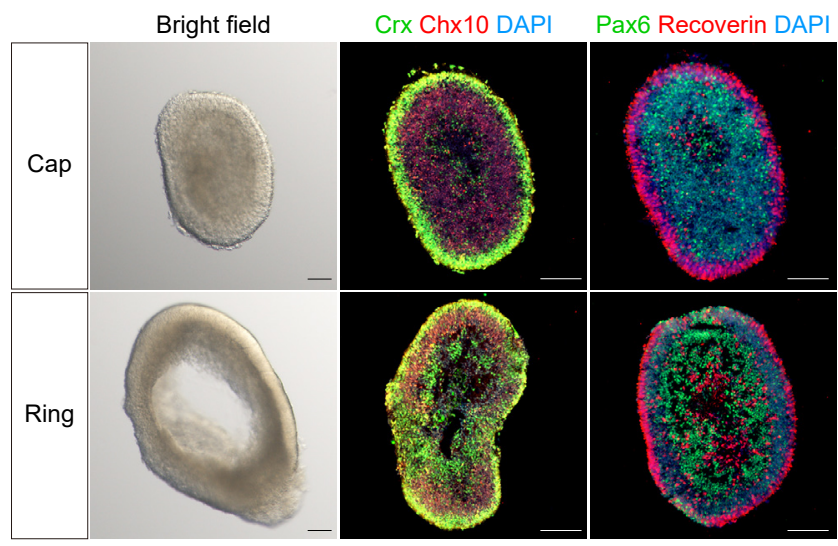

C

Gene expression analysis of Cap and Ring from various tissues

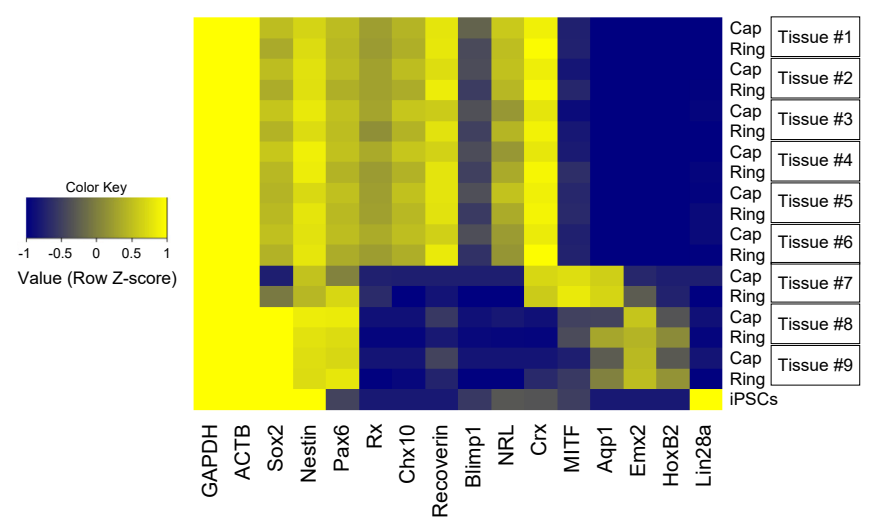

D

Correlation of mRNA levels of marker genes in cap and ring

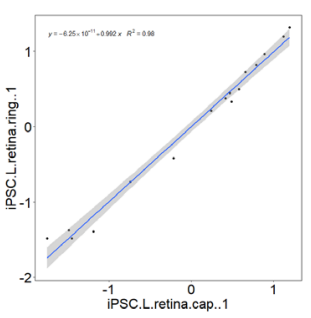

\begin{tabular}{cc}
\hline Sample & $\mathrm{R}^{2}$ \\
\hline$\# 1$ & 0.98 \\
$\# 2$ & 0.99 \\
$\# 3$ & 0.98 \\
$\# 4$ & 0.99 \\
$\# 5$ & 0.99 \\
$\# 6$ & 0.98 \\
\hline
\end{tabular}

Figure 3. QC method for retinal sheets by testing gene expression in the surrounding outer tissue-sheet.

(A) Scheme for dissection of the cap and the ring and the ring-PCR test in Process 5 (dissection).

(B) Bright-field (left) and IHC (middle, right) images of a representative cap and ring. The cap and ring were derived from iPSC-LPF11 cells. Immunostaining for Crx (middle, green), Chx10 (middle, red), Pax6 (right, green), and Recoverin (right, red) is shown. DAPI is shown in blue. Scale bar: $100 \mu \mathrm{m}$.

(C) Heat map for gene expressions in caps and rings derived from iPSC-LPF11 cells. As a control, gene expressions in undifferentiated iPSC-LPF11 cells are also shown. Gene expressions were measured by qPCR analyses. Row Z-scores were calculated for each cap and ring, and plotted as a heat map.

(D) Representative gene expressions in cap and ring dissected from the same aggregate shown as a plot with a regression line (left). The coefficient of determination $\left(\mathrm{R}^{2}\right)$ for the gene expression between each cap and ring is shown (right). \#1-\#6 correspond to Tissue \#1-\#6 in $(\mathrm{C})$. 
Screening for non-freezing preservation conditions

\begin{tabular}{lccc}
\hline & $4^{\circ} \mathrm{C}$ & $17^{\circ} \mathrm{C}$ & $37^{\circ} \mathrm{C}$ \\
\hline Maturation medium & - & ++ & +++ \\
UW solution & $-1+$ & - & - \\
BSS & + & ++ & + \\
Optisol-GS & + & +++ & ++ \\
\hline
\end{tabular}

C

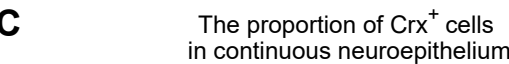

B

\begin{tabular}{ccc}
$\begin{array}{c}\text { iPSC-retina } \\
\mathrm{d} 0\end{array}$ & $\begin{array}{c}\text { Preserved retina } \\
\text { Recovered retina }\end{array}$ \\
\hline
\end{tabular}

D

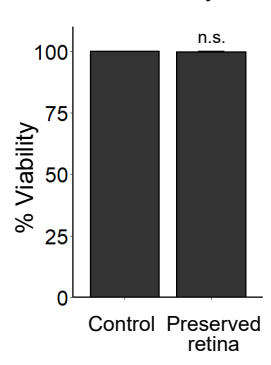

E

Chx10 mRNA

Crx mRNA
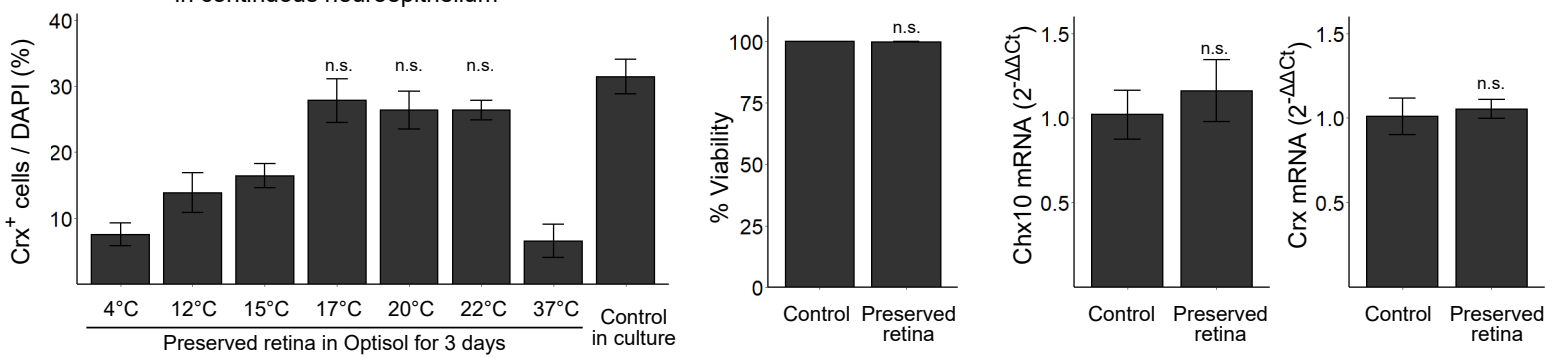

$\mathbf{F}$

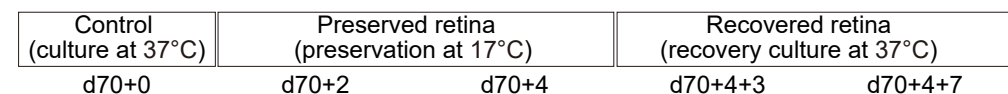

G The number of EdU ${ }^{+}$cells
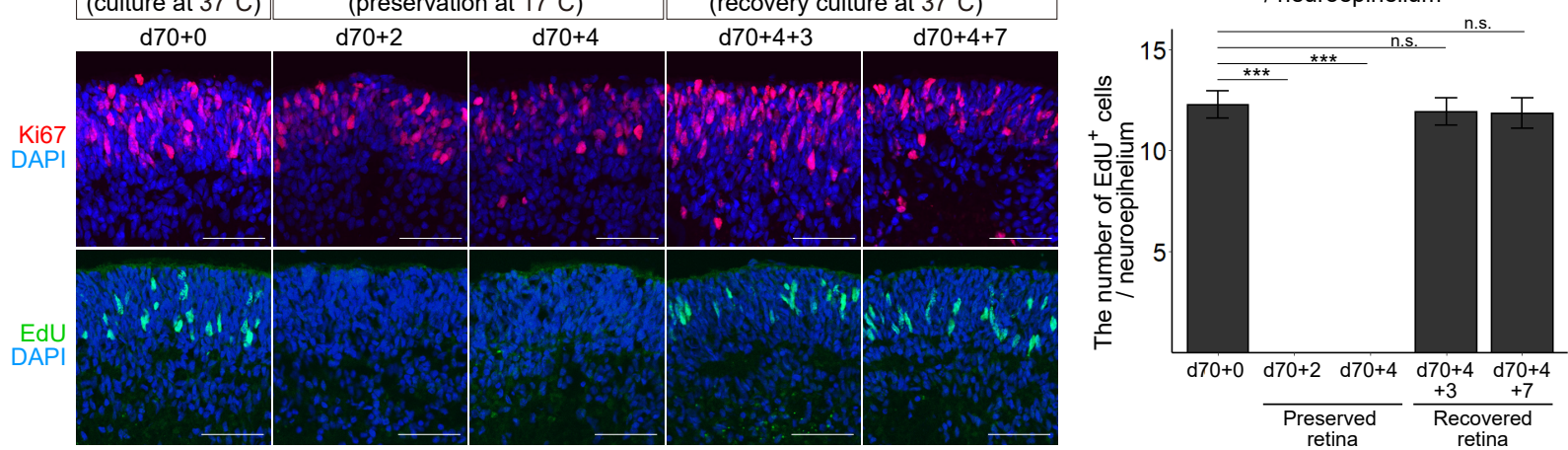

H

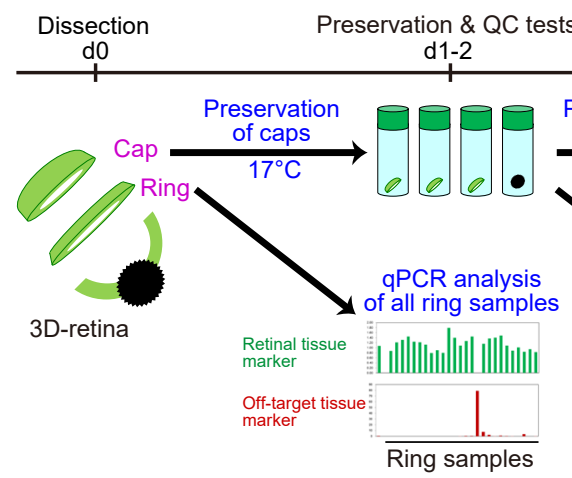

Release d2-3

Transplantation d3-4

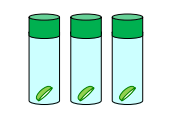

Retinal sheets (Passed caps)
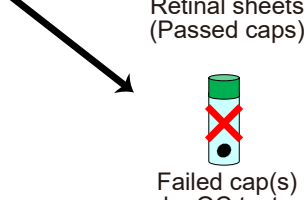

by QC tests

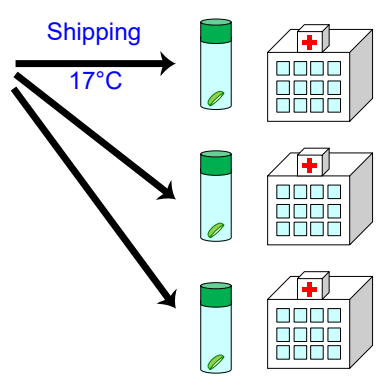

Figure 4. Controlled room temperature non-freezing preservation method.

(A) Screening for the non-freezing preservation conditions. +++: multilayered continuous retinal epithelium was preserved. ++: multilayered continuous retinal epithelium was preserved but neural rosettes appeared. +: multilayered continuous retinal epithelium was observed but very limited. -, no multilayered continuous retinal epithelium was observed after the preservation.

(B) Scheme of the preservation experiments. iPSC-retinas were preserved in Optisol at $17^{\circ} \mathrm{C}$ for $3-4$ days followed by recovery culture at $37^{\circ} \mathrm{C}$ under $\mathrm{CO}_{2}$ for 7 days. Recovered retinas were subjected to experiments.

(C) Proportions of $\mathrm{Crx}^{+}$photoreceptor precursors in multilayered retinal tissues. The proportions were calculated by dividing the number of $\mathrm{Crx}^{+}$cells by the number of nuclei (DAPI). Data are presented as mean $\pm \mathrm{SE}$ for $n=5\left(4{ }^{\circ} \mathrm{C}\right), 4\left(12{ }^{\circ} \mathrm{C}\right), 4\left(15^{\circ} \mathrm{C}\right), 5\left(17^{\circ} \mathrm{C}\right), 5\left(20^{\circ} \mathrm{C}\right), 5\left(22^{\circ} \mathrm{C}\right), 4\left(37^{\circ} \mathrm{C}\right)$, and 3 (control culture).

(D) Cell viability in non-preserved retinas (Control) and retinas preserved for 4 days. Data are shown as mean $\pm \mathrm{SE}(n=5$ per group).

(E) Gene expressions of Chx10 and Crx in non-preserved retinas (Control) and retinas preserved for 4 days, determined by qPCR analyses. Data are presented as mean $\pm \mathrm{SE}(n=3$ per group).

(F) IHC analysis of retinas that were not preserved (d70+0), preserved in Optisol for 2 days $(\mathrm{d} 70+2)$ and 4 days (d70+4), and preserved in Optisol for 4 days followed by recovery culture for 3 days $(\mathrm{d} 70+4+3)$ and 7 days $(\mathrm{d} 70+4+7)$. Ki67 (red) staining in the upper panels. EdU staining (green) in lower panels. DAPI is shown in blue. Scale bar: $50 \mu \mathrm{m}$.

(G) Numbers of EdU-positive cells per $100 \mu \mathrm{m}$-wide multilayered retinal tissue in each group. Data are presented as mean $\pm \mathrm{SE}(n=6$ per group). $* * * p<0.001$ for one-way ANOVA followed by a Tukey's test.

(H) Scheme for dissection, ring-PCR test, and shipping of retinal sheets. Dissected caps are preserved at $17{ }^{\circ} \mathrm{C}$. During the preservation, the corresponding rings are subjected to the ring-qPCR test. Caps whose rings pass the ring-PCR test can be shipped as retinal sheets for transplantation. n.s., not significant. 


\section{Figure 5}
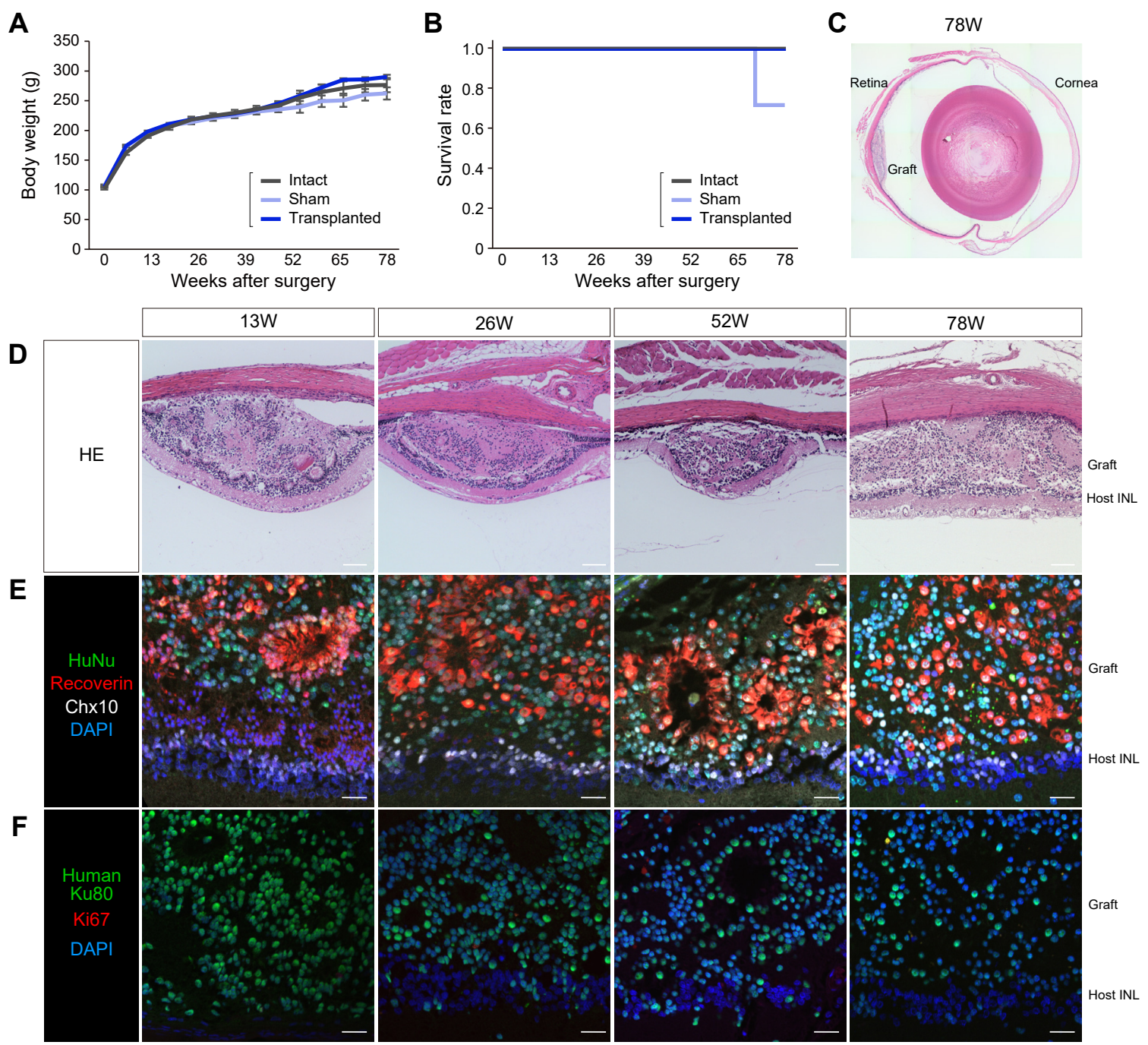

$\mathbf{G}$
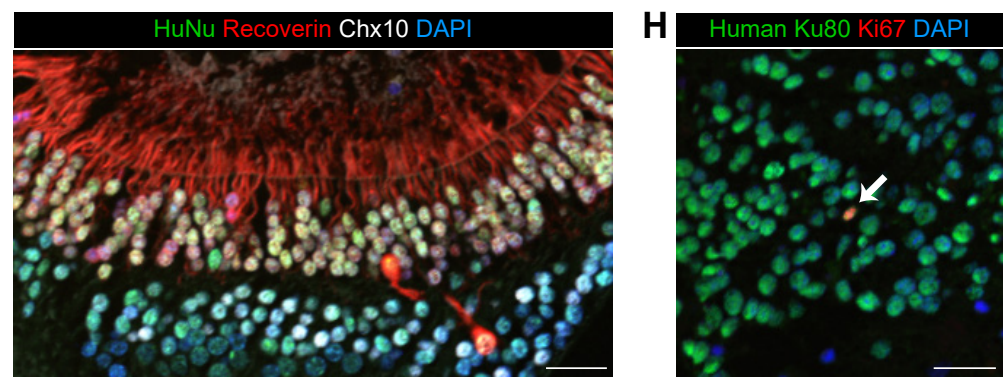

I

\begin{tabular}{lllll}
\cline { 2 - 4 } $\mathrm{Ki} 7^{+} \mathrm{Ku} 80^{+} / \mathrm{Ku} 80^{+}(\%)$ & $13 \mathrm{~W}$ & $26 \mathrm{~W}$ & $52 \mathrm{~W}$ & $78 \mathrm{~W}$ \\
\cline { 2 - 5 } & 0.32 & 0.36 & 0.11 & 0.65 \\
\hline
\end{tabular}

Figure 5. In vivo tumorigenicity study by subretinal transplantation in nude rats.

(A,B) Time courses of body weight (A) and Kaplan-Meier survival curves (B) in nude rats in the tumorigenicity study. Intact: control nude rat without surgery $(n=4)$. Sham: control nude rat with subretinal injection of vehicle control $(n=4)$. Transplanted: nude rat with subretinal transplantation of a single iPSC-Q-derived retinal sheet $(n=4)$. Data are presented as mean $\pm \mathrm{SE}$.

(C,D) HE staining of transplanted eye sections at 13, 26, 52, and 78 weeks after transplantation. Scale bar: $100 \mu \mathrm{m}$.

(E-I) Immunostaining of transplanted eye sections for human nuclear markers, retinal markers, and Ki67. Scale bar: $20 \mu \mathrm{m}$. (E) Immunostaining for HuNu (green), Recoverin (red), Chx10 (white), and DAPI (blue). (F) Immunostaining for human Ku80 (green), Ki67 (red), and DAPI (blue). (G) High-magnification image of immunostaining for a graft in the vitreous cavity (52 weeks). HuNu (green), Recoverin (red), Chx10 (white), and DAPI (blue). (H) High-magnification image of immunostaining for the graft (52 weeks) with antibodies for human Ku80 (green), Ki67 (red), and DAPI (blue). Arrow indicates Ki67 and Ku $80^{+}$cells. (I) Percentages of Ki67 $7^{+}$and $\mathrm{Ku} 80^{+}$cells among the Ku80 human cells. Data are presented as mean. INL, inner nuclear layer. 


\section{Figure 6}
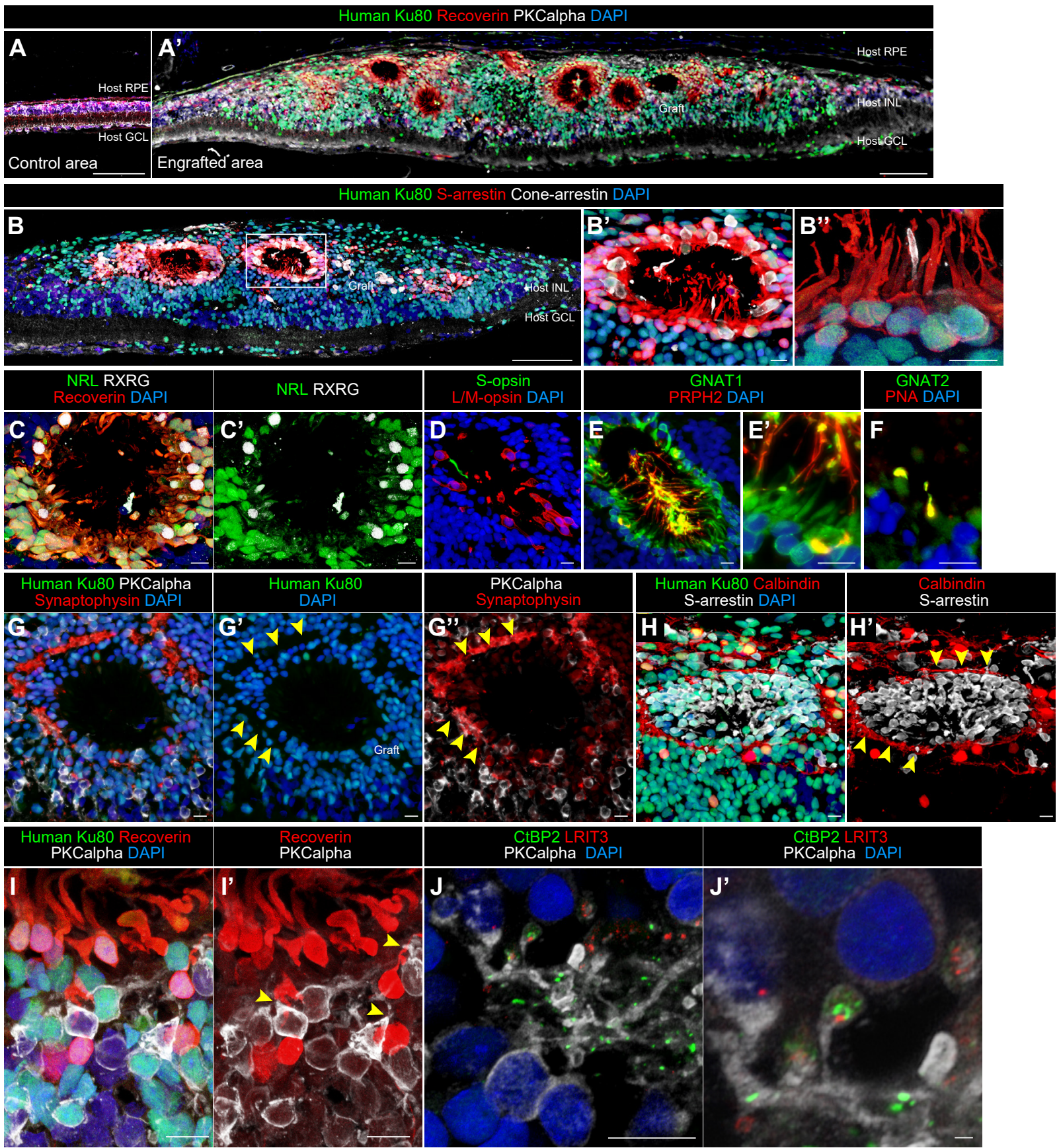

Figure 6. Engraftment and photoreceptor maturation of iPSC-retinal sheets after subretinal transplantation in RD-nude rats.

(A-J') Immunostaining of rat eyes transplanted with iPSC-S17-derived retinal sheets. The retinal sheets were transplanted in the subretinal space of RD-nude rats. The rat retinas were fixed at 263 days after transplantation (341 days after initiation of differentiation).

(A,A') Immunostaining for human Ku80 (green), Recoverin (red), and PKCalpha (white). Control non-transplanted area (A). Engrafted area (A'). (B-B") Immunostaining for human Ku80 (green), S-arrestin (red), and Cone-arrestin (white). High magnification in (B') and higher magnification in (B"). Boxed area in (B) corresponds to (B').

(C-I') Immunostaining for photoreceptor markers. NRL (green), Recoverin (red), and RXRG (white) in (C,C'). S-opsin (green) and L/M-opsin (red) in (D). GNAT1 (green) and PRPH2 (red) in (E,E'). GNAT2 (green) and PNA (red) in (F). Human Ku80 (green), Synaptophysin (red), and PKCalpha (white) in $\left(\mathrm{G}-\mathrm{G}^{\prime \prime}\right)$. Arrowheads in $\left(\mathrm{G}^{\prime}, \mathrm{G}^{\prime \prime}\right)$ : Synaptophysin-positive neurites in no nuclear space. Human Ku80 (green), Calbindin (red), and S-arrestin (white) in (H,H'). Arrowheads in (H'): Calbindin-positive neurites.

(I,I') Maximum projection image of Z-stacks immunostained for human Ku80 (green), Recoverin (red), and PKCalpha (white) in (I,I'). Note that human $\mathrm{Ku} 80^{-}$and $\mathrm{PKCalpha}{ }^{+}$bipolar cells were located near the human $\mathrm{Ku} 80^{+}$and Recoverin ${ }^{+}$photoreceptors (arrowheads).

(J,J') Maximum projection image of Z-stacks stained for CtBP2 (green), LRIT3 (red), and PKCalpha (white). High magnification in (J'). Note that photoreceptor-synapse marker CtBP2 and LRIT3 were expressed near the neurites of PKCalpha ${ }^{+}$rod bipolar cells.

DAPI staining (blue) in (A,A',B-B',C,D,E,E',F,G,G',H,I,J,J'). Scale bars: $100 \mu \mathrm{m}$ in (A',B), $10 \mu \mathrm{m}$ in (A,B',B”,C-J), and $1 \mu \mathrm{m}$ in (J').

$\mathrm{INL}$, inner nuclear layer; GCL, ganglion cell layer. 


\section{Figure 7}

A

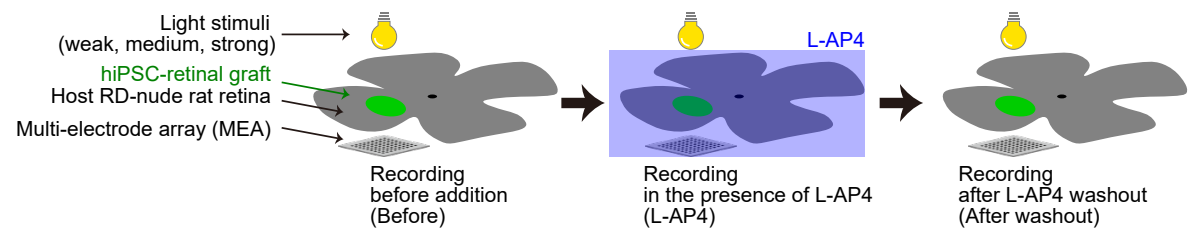

B

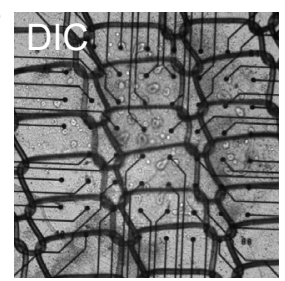

C

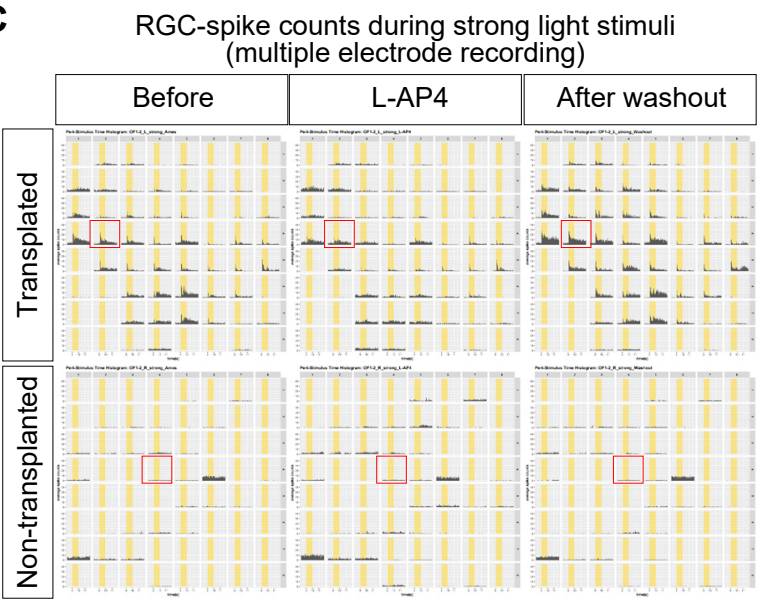

D

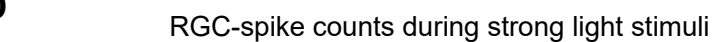

$\mathbf{E}$
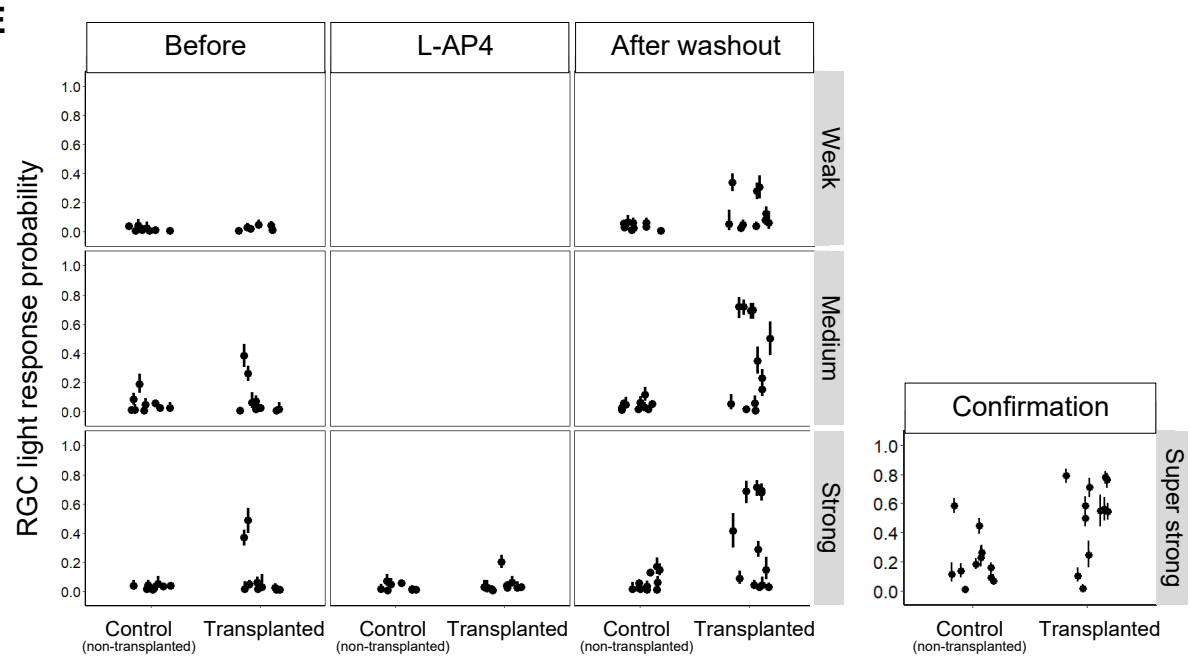

RGC light response probability

in each recorded retina

(Control [ $n=12]$, Transplanted [ $n=12]$ )

Figure 7. Light responses in transplanted iPSC-retinas by ex vivo electrophysiology assay.

(A) Scheme of ex vivo electrophysiology assay using MEA to evaluate the light responses in transplanted iPSC-retinas. iPSC-S17-derived retinal sheets were transplanted in the subretinal space in RD-nude rats. Transplanted and non-transplanted rat retinas were mounted on the MEA electrodes for electrophysiological recording. During the recording, light stimuli were conducted in different intensities (weak, medium, strong), and the rat retinas were incubated in Ames' medium (Before), followed by addition of L-AP4 (L-AP4), and washout of L-AP4 (After washout).

(B-D) Representative results of MEA analysis.

(B) Differential interference contrast (DIC) image of a RD-nude rat retina mounted on the MEA.

(C,D) Peri-stimulus time histograms to examine the RGC-spike counts during strong light stimuli $\left(12.84 \log\right.$ photons $\left./ \mathrm{cm}^{2} / \mathrm{s}\right)$. The $\mathrm{X}$-axis and $\mathrm{Y}$-axis represent the time and average RGC-spike count, respectively. Recording data for iPSC-retinal sheet-transplanted rat retinas (Transplanted; upper) and non-transplanted rat retinas (Non-transplanted; lower) in the three conditions (Before, L-AP4, After washout) are shown. Multiple electrode recording data are shown in (C) and representative data (red box in C) are shown in (D). Yellow box in (C,D): light stimuli. Red arrows in (D): start timing of light stimuli.

(E) RGC light response probabilities. Control non-transplanted rat retinas (Control; $n=12$ ) and iPSC-S17-derived retinal sheet-transplanted rat retinas (Transplanted; $n=12$ ) were subjected to MEA analysis to evaluate the RGC responses to light stimulation. Dots and bars show the per sample (recorded rat retina) summary of the collected data. Dot: RGC light response probability estimated by statistical modeling (Bayesian statistical inference with Markov Chain Monte Carlo sampling). Bar: 89\% confidence interval. 